\title{
Workload capacity spaces: A unified methodology for response time measures of efficiency as workload is varied
}

\author{
James T. Townsend • Ami Eidels
}

Published online: 24 May 2011

(C) Psychonomic Society, Inc. 2011

\begin{abstract}
Increasing the number of available sources of information may impair or facilitate performance, depending on the capacity of the processing system. Tests performed on response time distributions are proving to be useful tools in determining the workload capacity (as well as other properties) of cognitive systems. In this article, we develop a framework and relevant mathematical formulae that represent different capacity assays (Miller's race model bound, Grice's bound, and Townsend's capacity coefficient) in the same space. The new space allows a direct comparison between the distinct bounds and the capacity coefficient values and helps explicate the relationships among the different measures. An analogous common space is proposed for the AND paradigm, relating the capacity index to the Colonius-Vorberg bounds. We illustrate the effectiveness of the unified spaces by presenting data from two simulated models (standard parallel, coactive) and a prototypical visual detection experiment. A conversion table for the unified spaces is provided.
\end{abstract}

Keywords Capacity · Workload - Race model · Coactive model $\cdot$ Response times $\cdot$ OR task $\cdot$ AND task

Imagine that you are the pilot of a commercial aircraft, full of passengers. Your warning system can provide a visual (red light) and an auditory (loud beep sound) signal to alert

J. T. Townsend Indiana University,

Bloomington, IN, USA

A. Eidels $(\bowtie)$

School of Psychology, University of Newcastle, Callaghan, NSW, Australia

e-mail: Ami.Eidels@newcastle.edu.au you to low altitude. Upon detection of either warning signal, you must respond immediately by pulling up and regaining altitude. Clearly, a quick response is vital. Would you respond to the threat faster in the presence of both warning signals, as compared with just one? Observe that in providing two rather than one signal, the workload on the perceiving system has increased. ${ }^{1}$ How does this affect the speed of responding? Because either signal can produce a correct response, we call this an $O R$ paradigm.

On the other hand, many situations necessitate perceiving two or more signals, whether distinct modalities or simply features of a pattern. For example, before the final approach for landing, the same pilot must receive auditory confirmation from air-traffic control and visually confirm that there is no other aircraft on the lane. Thus, the pilot must complete processing of two sources of information, which by itself adds time to the processing duration. Again, the increase in workload, from one to two, also can affect response times (RTs). Due to the requirement of completing two signals, we call this an $A N D$ paradigm. As we will detail subsequently, in order to assess the internal efficiency in processing, it is critical to take into account the decisional stopping rule (e.g., OR vs. AND) that is being

\footnotetext{
${ }^{1}$ An increment in workload is usually natural to define in terms of number of letters, dimensions, or subtasks involved in some task. We shall often refer simply to items or, sometimes, channels as generic tags for the discrete objects being processed or the conduits working on them. The unit of workload typically relates in a natural fashion to the task, so that ultimate definitions of, for instance, feature, are unnecessary. Thus, if a search task involves examination of a list of letters, the unit may fairly be made in terms of letters. Other conditions might involve operationally defined features (e.g., lines, angles, curves) or dimensions such as color, size, and the like.
} 
used by the participant. In fact, the decisional rule itself tends to increase or decrease RTs as the workload is varied, with no other changes in architecture or individual-channel efficiency.

For well over a hundred years, mean RT has been the preferred statistic with which to measure efficiency (although accuracy runs a close second). In this article, we review recent progress and propose a method of unifying various statistics of efficiency that afford depiction in a single space using a common scale. Two small experiments were run to yield data that we use to exemplify the new scaling structures.

Over the last few decades, mathematical characterization of mental processes has helped to refine our notions regarding how RTs relate to the fundamental ability of a system to deal with heavier task duties (Kahneman, 1973; Townsend \& Ashby, 1978, 1983). We informally refer to this ability as efficiency or, more technically, as workload capacity. ${ }^{2}$ A number of statistics besides mean RT are now being used to provide more precise assays of capacity. Townsend and Ashby (1978) dealt with many aspects of capacity and suggested that hazard functions (defined below) offer a highly articulated way of measuring it. Around the same time, J. Miller $(1978,1982)$ suggested an upper bound on RTs for channels involved in a race within a redundant-target paradigm. In such a design, which calls for an inclusive-disjunctive paradigm, or simply an OR paradigm (since the participant is instructed to detect the presence of a particular target item, or another target item, or both), any target item leads to a correct response and, hence, prompts a race in a parallel system. Likewise, Grice and colleagues proposed a lower bound on performance in such systems (e.g., Grice, Canham, \& Gwynne, 1984). We subsequently have worked out a general theory of workload capacity based on a type of statistic called $C_{\mathrm{OR}}(t)$ and linked it both to Grice's and Miller's race model bounds (Townsend \& Nozawa, 1995).

More recent efforts along these lines expanded the theory to include tasks that demand processing of all presented items (Townsend \& Wenger, 2004b). We refer to the latter as $A N D$ (also termed conjunctive) designs because

\footnotetext{
${ }^{2}$ The term capacity is used elsewhere for different purposes-for example, in information theory, to indicate the number of bits a system or a channel transmits per second under optimal coding (e.g., Shannon, 1948; Shannon \& Weaver, 1949), and in memory research, to indicate the number of items that can be stored in short-term or working memory (e.g., Baddeley, 2001; Baddeley \& Hitch, 1974; Cowan, 2005; G. A. Miller, 1956). We confine our definition of the term capacity to the relative change in speed by which the cognitive system processes information as we increase the workload by increasing the amount of to-be-processed information.
}

processing must generally be applied to all items in order for a correct response to occur (processing is often said to be exhaustive in such cases). Because assays of capacity depend on the stopping rule (OR requires only firstterminating or minimum time processing, whereas AND requires maximum finishing time or last-terminating), we developed an appropriate capacity measure for this case, called $C_{\mathrm{AND}}(t)$ (when we present the formula for this measure we shall explain why it is necessary to employ different capacity measures for the OR and AND cases). Colonius and Vorberg (1994) had put forth upper and lower bounds appropriate for AND designs, analogous to those of Miller's and Grice's in the OR paradigm, and our theory encompasses those and connects them to $C_{\mathrm{AND}}(t)$.

Other properties of the human information-processing system are also important in determining the efficiency and speed with which we process multiple signals. Thus, the architecture of the attendant processes - that is, the arrangement of subprocesses (such as, serial vs. parallel arrangement)-also affects the efficiency of a system as the number of subtasks increases. The decisional stopping rule (stopping rule for short), which determines how much information a system processes before ceasing, as noted above, can also play a major role in efficiency assessment. Finally, potential correlations, the so-called dependence issue, across subtasks, channels, or items, can impact capacity measurements. The foundations of these issues are developed in a series of papers and books (e.g., Townsend, 1972, 1974, 1990; Townsend \& Ashby, 1983; Townsend \& Wenger, 2004a) and are outlined here only as needed.

Formerly, measures proposed by various investigators have had to be computed and plotted in different scales, or spaces, which render comparisons challenging if not impossible. In one case, the measurements are plotted as probabilities, whereas in the other, they are plotted as ratios of transformations of probabilities. (More technical descriptions will follow below when the requisite machinery has been provided.). Our general goal here is to propose unified workload capacity spaces, which we designate $C_{\mathrm{OR}}$ space and $C_{\mathrm{AND}}$ space, for the OR and the AND designs, respectively, and a set of strategies for portraying a number of important statistics within each space. Furthermore, we provide detailed formulae for transforming data at the different levels and bounds into statistics that can be immediately plotted in the new spaces. Anyone interested in measuring a system's capacity and the efficiency of processing can use the convenient summary in Table 1 to obtain formulae for estimating and plotting, on a single figure, the various statistics from empirical data (collected in the appropriate multiple-target task, often termed the redundant-target task, which we outline below). 
Table 1 Summary of the pertinent tests in their conventional and novel forms. The latter is a transformation onto the capacity coefficient space ( $C$ space, in short). The three top rows correspond to an OR experiment, whereas the bottom three rows correspond to an
AND experiment. $C(t)$ is expressed in terms of $F(t)$ and $S(t)$, rather than $H(t)$ and $K(t)$, to allow convenient estimation from experimental data

\begin{tabular}{|c|c|c|}
\hline Test & Conventional Form & Form on $C$ Space \\
\hline $\begin{array}{l}\text { Capacity coefficient- OR task } \\
\text { (Townsend \& Nozawa, 1995) }\end{array}$ & & $C_{O R}(t)=\frac{\log \left[S_{A B}(t)\right]}{\log \left[S_{A}(t) \cdot S_{B}(t)\right]}$ \\
\hline $\begin{array}{l}\text { Race model inequality } \\
\text { (J. Miller, 1982) }\end{array}$ & $F_{A B}(t) \leq F_{A}(t)+F_{B}(t)$ & $C_{O R}(t) \leq \frac{\log \left[S_{A}(t)+S_{B}(t)-1\right]}{\log \left[S_{A}(t) \cdot S_{B}(t)\right]}$ \\
\hline $\begin{array}{l}\text { Grice inequality } \\
\text { (Grice et al., 1984) }\end{array}$ & $F_{A B}(t) \geq M A X\left[F_{A}(t), F_{B}(t)\right]$ & $C_{O R}(t) \geq \frac{\log \left\{M I N\left[S_{A}(t), S_{B}(t)\right]\right\}}{\log \left[S_{A}(t) \cdot S_{B}(t)\right]}$ \\
\hline $\begin{array}{l}\text { Capacity coefficient-AND task } \\
\text { (Townsend \& Wenger, 2004b) }\end{array}$ & & $C_{A N D}(t)=\frac{\log \left[F_{A}(t) \cdot F_{B}(t)\right]}{\log \left[F_{A B}(t)\right]}$ \\
\hline $\begin{array}{l}\text { Colonius-Vorberg } \\
\text { upper bound (1994) }\end{array}$ & $F_{A B}(t) \leq M I N\left[F_{A}(t), F_{B}(t)\right]$ & $C_{A N D}(t) \leq \frac{\log \left[F_{A}(t) \cdot F_{B}(t)\right]}{\log \left\{M I N\left[F_{A}(t), F_{B}(t)\right]\right\}}$ \\
\hline $\begin{array}{l}\text { Colonius-Vorberg } \\
\text { lower bound (1994) }\end{array}$ & $F_{A B}(t) \geq F_{A}(t)+F_{B}(t)-1$ & $C_{A N D}(t) \geq \frac{\log \left[F_{A}(t) \cdot F_{B}(t)\right]}{\log \left[F_{A}(t)+F_{B}(t)-1\right]}$ \\
\hline
\end{tabular}

The next section provides an outline of some aspects of capacity measures' relationships under different experimental circumstances. It is mildly more technical than the other sections and can likely be skipped on a first reading.

\section{Measures of capacity and their interrelationships ${ }^{3}$}

Static and single-signal versus multiple-target capacity measures

Efficiency of processing can be measured either under experimentally static conditions or with variations in the effort required by the participants. In the former, the experimenter may well be interested in the way in which efficiency changes across time, rather than across conditions such as workload. Machinery is required for both situations. Originally, we employed the term capacity to refer to a number of quantitative measures of efficiency, especially when collecting RTs. In fact, our results, theoretical and experimental, started using the term capacity from the beginning (e.g., Townsend, 1972 and, especially, 1974).

As the methodology has evolved, it has become clear that there is a need to distinguish the static, with regard to task effort, versus the alteration of workload. Of course, we often see the same tools in both but used and analyzed in a rather different manner. Thus, the Townsend and Ashby (1978) theory proposed the hazard function (see below for rigorous

\footnotetext{
$\overline{3}$ In what follows, we assume that our probability distributions always possess densities.
}

treatment) as a potentially effective measuring instrument of efficiency, which they generically called capacity. The hazard function has been employed since then frequently as a labor-static design (not to exclude the possibility that a participant might tire or warm up during a session, but the experimenter is not so manipulating the task). In addition, certain powerful stochastic methods have recently been enlisted that considerably enhance the utility and statistical sensitivity of hazard function analyses with psychological data (Chechile, 2003; Wenger \& Gibson, 2004).

Here, we propose to refer to a measurement of efficiency made in a situation where the effort is manipulated as workload capacity. Correspondingly, we suggest using capacity either in a static domain or, after so advising the audience, as a generic concept. From here on out, we focus on workload capacity.

The hazard and integrated hazard functions

The hazard function gives the instantaneous rate of completion at any point in time, given that the process under observation has not yet completed. In a stochastic renewal process, it can be taken as the rate of performing work in terms of number of completions per unit time (e.g., Parzen, 1962, pp. 168-169).

The integral of the hazard function up to an arbitrary time $t$ can analogously be interpreted as the amount of work done, or energy expended, in units of the to-be-completed items or channels. Both the hazard and the integrated hazard function are valid indicators of efficiency, but the hazard function is naturally a more fine-grained measurement than its integral, just as power is more fine grained than its integral energy expended in physics. 
In principle, the hazard function could be used in workload capacity measures (in the same type of ratios as derived for integrated hazard functions) but hazard functions are challenging to estimate, despite the advances in this technology (e.g., Wenger \& Gibson, 2004). Our experience has been that the integrated hazard function is a more stable statistic, since integrated functions often are, and a rough and ready estimate is immediately supplied by taking the logarithm of the survivor function.

The yardstick: standard parallel process models

Statistics such as the mean RT, the hazard function, or others can be employed to assess speed of processing. However, to purchase theoretical authority in the sense of model discrimination, we need to formulate some benchmark or yardstick of comparison. Such a yardstick affords the ability to assess workload capacity across differing systems by employing such statistics as the hazard function in a nonparametric way.

The choice of the yardstick measuring device and, in particular, the architecture classification are, to some extent, simply a matter of preference and convention. As our measuring instrument, we have settled on systems with independent, parallel channels whose individual-channel processing characteristics do not change as the workload is varied. We refer to this model type as the standard parallel model (e.g., Townsend \& Wenger, 2004a). Aside from personal preference, the following seems to be a reasonable claim: In the fields of study where quantitative modeling of RTs has been most advanced, much interest has centered on whether performance is sufficiently fast to merit the term parallel processing or, alternatively, whether performance may be even faster than ordinary parallel processing. Visual search, attention, and short-term memory search are examples of the first theme, while redundant-signals perception forms a prime exemplar of the second theme. There may turn out to be some special benefit to applying a different architecture as the yardstick — say, serial processing — but that remains to be seen. This issue is discussed further in the Agreeing on a Taxonomy of Psychological Systems subsection in the Discussion section.

Finally, it is important to note that the two distinct capacity measures we shall soon employ, for OR and AND tasks, are expressed relatively to the performance of the same standard parallel model described above.

\section{Workload capacity for differing decision rules}

Consider a proposition put forward in terms of the predicate calculus. The proposition can be presumed to be a logical concatenation of constituent atomic statements, each of which is true or false (e.g., present vs. absent and so on).
The experimenter requires the participant to make a binary response on the basis of the proposition, for instance, its overall truth or falsity. Thus, the famous Sternberg task (1966) is of this nature where the participant adjudges whether the statement "a target item is contained in the memory set" is true or not.

Any stimulus, so constituted, can be answered by establishing the truth or falsity of a finite number of queries - for example, items or channels completed. The two extremes are found in OR and AND designs where the first item completed provides an answer versus all items must be finished, respectively. In order to lessen confusion, we will treat the OR and AND experimental tasks and pertinent measures in separate sections.

\section{Redundant-target (OR) task}

The capacity functions can be brought into play whenever a clearly expressible workload is varied and RTs are measured. Up to now, they have primarily been applied in redundanttarget search tasks. In such tasks, the participant attempts to detect whether one or more operationally defined targets are present (visual, acoustic, or tactile stimuli, etc.). Although the details of the design differ, all redundant-target paradigms include a subset of trials on which multiple targets appear. Redundant-target trials prima facie permit OR responding, since the perception of any of the targets can legitimately cause a cessation of processing. We will deal with AND trials subsequently. Hereon, we write as if all signals are visual, but of course, this need not be the case.

In a visual redundant-target detection task, targets may be presented in one location (say, A), in another (B), in both (AB; the double-target display), or in neither. On the latter type of trials, which are often called target-absent trials, targets do not appear at all. Participants are asked to respond affirmatively if they detect the presence of at least one target (i.e., target $\mathrm{A}$ alone, $\mathrm{B}$ alone, or $\mathrm{AB}$ ), and respond negatively or withhold response otherwise (i.e., if no target was present). When a single target is present, A alone, the cumulative distribution function (also known as the cumulative frequency function, especially when data are considered) is expressed as $P\left(\mathrm{RT}_{\mathrm{A}} \leq t\right)=F_{\mathrm{A}}(t)$ and similarly for $\mathrm{B}$. When both locations are occupied by targets, we write $P\left(\mathrm{RT}_{\mathrm{AB}} \leq t\right)=F_{\mathrm{AB}}(t)$. In many experiments, trials with redundant targets randomly alternate with those containing single targets (and those with no target at all) on a within-block basis.

The measures we discuss below are all based on RTs collected in redundant-target tasks, and use correct responses only. In many redundant-target studies that focus on RTs, including the experiments reported here, error rates are sufficiently low to be safely ignored. When error rates are high enough, other measures of performance can be 
brought into play, which focus on the pattern of errors rather than on RTs. ${ }^{4}$

\section{Miller's race model inequality (OR tasks)}

It will be necessary to begin employing theoretical terms. Some, like the race model inequality, are immediately susceptible to formal quantitative meaning. Others will be offered first informally and later technically.

J. Miller $(1978,1982)$ proposed an upper bound for performance on double-target trials - if the two targets are racing and the winner determines the processing time- the race model inequality:

$F_{A B}(t) \leq F_{A}(t)+F_{B}(t)$

The inequality states that the cumulative distribution function for the double-target display, $F_{\mathrm{AB}}(t)$, cannot exceed the sum of the single-target cumulative distribution functions, if processing is, as J. Miller (1982) expressed it, an ordinary race. The term race is generally interpreted as occurring between parallel channels. Although maintaining this convention, it should not be forgotten that other architectures, such as serial, might be theoretical candidates in many cognitive situations. Serial models generally obey the above race inequality, but there exist plausible situations where they do not (Townsend \& Nozawa, 1997).

Now consider the strategic model class that is formed from parallel models that possess stochastically independent channels and whose individual channels run just as fast, but no faster, when other channels in the system are operating, the condition of unlimited capacity (which we define formally in the next section, Capacity Coefficient). We referred to this model type earlier as the standard parallel model.

Any standard parallel model, with a first-terminating stopping rule (i.e., "stop as soon as any target is found," implying a minimum time statistic), makes the prediction

\footnotetext{
${ }^{4}$ Performance measures that are based on patterns of error rates are beyond the scope of this article. They become particularly valuable when stimuli are presented near threshold, in low contrast, and/or via the addition of external noise (e.g., Lu \& Dosher, 1998). We mention three accuracy-based approaches that seem especially propitious for search paradigms. The first has been taken by J. Palmer (e.g., Palmer, Verghese, \& Pavel, 2000). Among other important facets, it has shown low-to-moderate-accuracy experimental situations exist that can be well-explained by unlimited capacity parallel processing. A second type of modeling was undertaken by Townsend and associates, which assumed a high threshold type of processing in serial or parallel models along with an appropriate guessing strategy (e.g., Busey \& Townsend, 2001; Townsend, 1981). A third approach has been employed by M. Shaw, who constructed an accuracy-based factorial method analogous to those used with RTs by various researchers to assess architecture (Mulligan \& Shaw, 1980; Shaw, 1982; and more recently, Eidels \& Townsend, 2009).
}

$\mathrm{F}_{\mathrm{AB}}(t) \leq F_{\mathrm{A}}(t \mid \mathrm{A}, \mathrm{B})+F_{\mathrm{B}}(t \mid \mathrm{A}, \mathrm{B})$, where on the right-hand side, the conditional notation $(\mathrm{A}, \mathrm{B})$ indicates the presence of both targets, as opposed to the single-target trials [e.g., $\left.F_{\mathrm{B}}(t \mid \mathrm{B})\right]$, and the subscript indicates which channel is being considered. If it is the case that $F_{\mathrm{A}}(t \mid \mathrm{A}, \mathrm{B})=F_{\mathrm{A}}(t \mid \mathrm{A})$ and $F_{\mathrm{B}}(t \mid \mathrm{A}, \mathrm{B})=\mathrm{F}_{\mathrm{B}}(t \mid \mathrm{B})$, an assumption known as context invariance which is tantamount to unlimited capacity, then Inequality 1 will follow. ${ }^{5}$ If the system is not unlimited capacity, it may be limited capacity or super capacity (e.g., Townsend \& Nozawa, 1995). If processing is limited capacity, it will satisfy the race inequality. Since the race inequality forms an upper bound, a super capacity model may, but need not, produce RTs that violate the bound (e.g., Townsend \& Wenger, 2004b).

At this stage in the development, we need to introduce the notion of coactivation. A system is coactive if the channels are parallel but, rather than each channel making a decision, the information from each channel is coalesced, usually summed, into a final conduit which then makes a decision about the presence of a target (Colonius \& Townsend, 1997; J. Miller, 1982; see also Townsend \& Wenger, 2004b, for a general formal treatment and references to a variety of studies on this topic). Hence, although parallel, there is no race, since the channels do not make individual decisions. By convention, the speeds of the individual channels do not change when more signals are added. The result is strong super capacity that always violates the race bound (Townsend \& Wenger, 2004b).

In the top left panel of Fig. 1, we illustrate the race model bound against the prediction of a standard parallel model (the model specifications are discussed in greater detail in the Model Predictions for OR Paradigm-Standard Parallel Model section). The solid lines with circle or triangle markers correspond to the cumulative distribution functions for the two single-target conditions. The dotted line represents the sum of the two single-target distributions at each time $t$-the race model bound. The inequality is satisfied as long as the observed distribution of the doubletarget condition (thick solid line without any markers) stays below the dotted line.

J. Miller (1982) argued that violations of the inequality allow rejection of all race models and may, consequently, be taken as evidence in favor of coactivation. ${ }^{6}$ If one takes as a defining characteristic of a race that it be parallel and

\footnotetext{
$\overline{{ }^{5} \text { Luce (1986) }}$ and Ashby and Townsend (1986) discuss these conditions in greater detail.

${ }^{6}$ As with many useful concepts in psychology, the concept of coactivation started out defined in a qualitative manner-for instance, operationally as a system that violated the race model inequality. Over the years, it became more rigorously and mathematically defined (e.g., Colonius \& Townsend, 1997; Diederich \& Colonius, 1991; J. Miller \& Ulrich, 2003; Schwarz, 1994; Townsend \& Nozawa, 1995).
} 
Fig. 1 Predictions of a standard parallel model with two independent and parallel channels and unlimited capacity (top row) and a coactive model (bottom row), in the OR task. The conventional form of the race bound is presented on the left panels as the sum of the singletarget cumulative distribution functions. Grice bound is given by the cumulative distribution function of the faster single target (B in this illustration). In the right panels, we present the same bounds transformed onto the capacity coefficient space

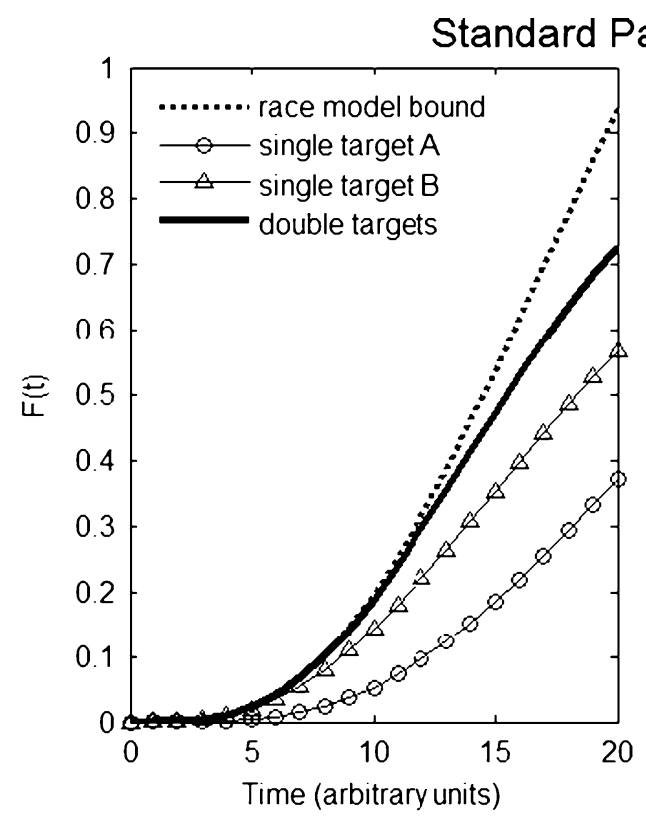

Standard Parallel Model

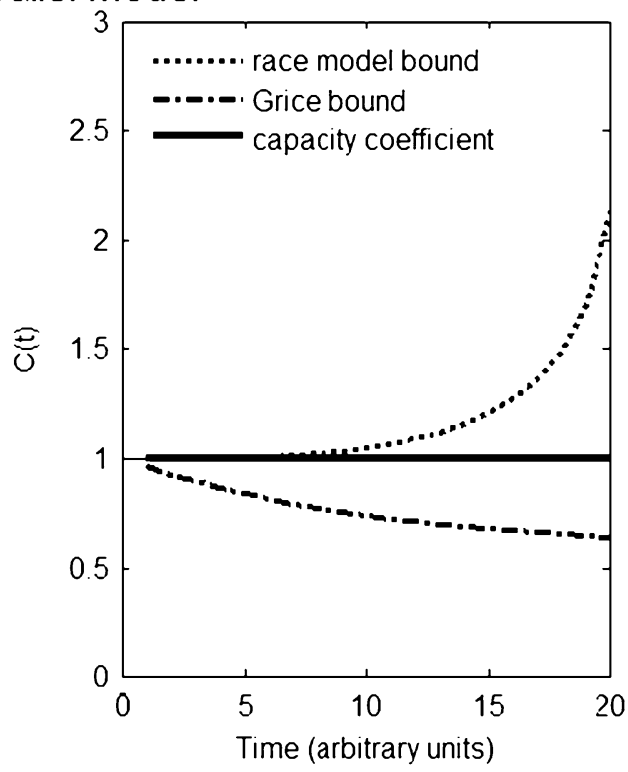

Coactive Model

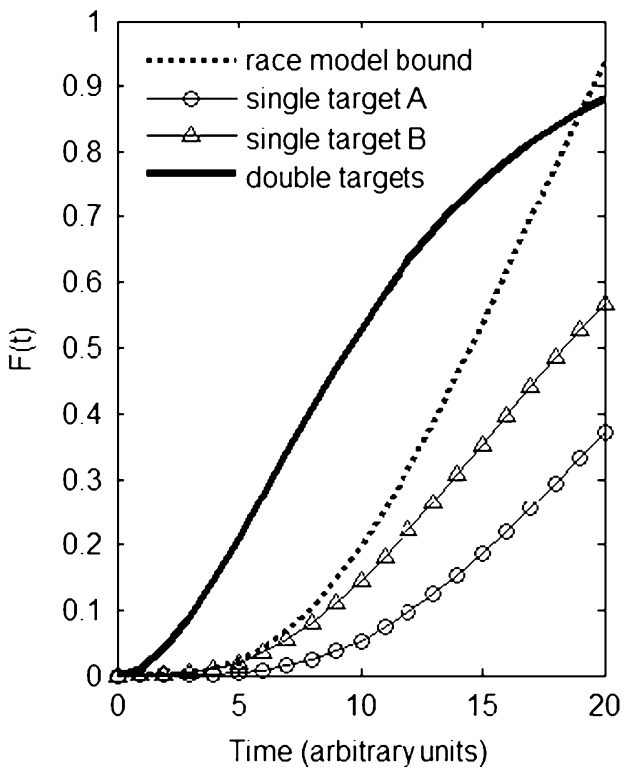

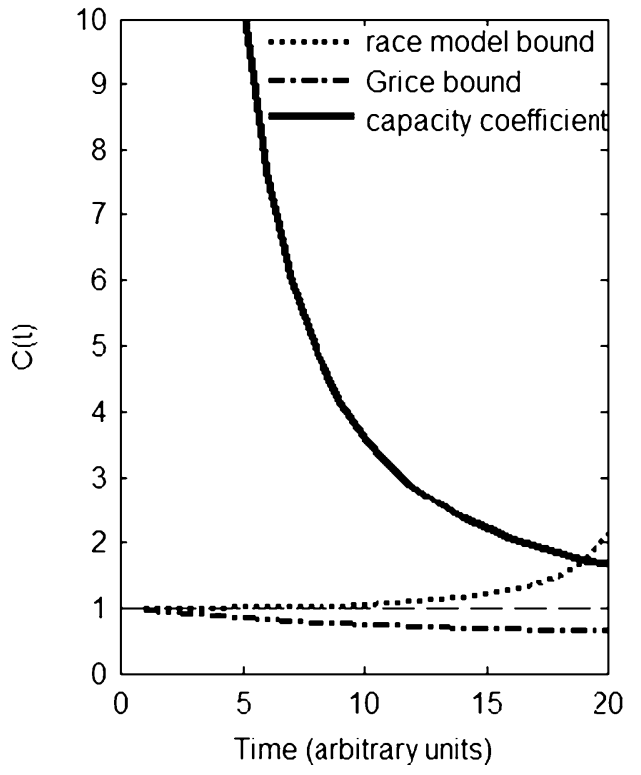

that the first channel to finish determines the processing time, then we would have to differ. The reason is that we have shown that race models in this sense, but which possess mutually facilitatory interchannel interactions, can readily predict violations of the race inequality (Townsend $\&$ Wenger, 2004b). The positive interchannel interactions defeat context invariance and cause super capacity (Eidels, Houpt, Altieri, Pei, \& Townsend, 2011). These models thereby form a class of systems that are super capacity but not coactive.

Now we are in a position to offer rigorous quantitative definitions of our workload capacity measure and the terms limited-, unlimited-, and super-capacity.
Capacity coefficient (OR tasks)

Townsend and Nozawa (1995) proposed an assay of performance on double-target trials - the capacity coefficientwhich is the ratio of the integrated hazard function of the double-target condition to the sum of the integrated hazard functions of the single-target conditions:

$C_{O R}(t)=\frac{H_{A B}(t)}{H_{A}(t)+H_{B}(t)}$

Here, we have defined the survivor function as the complement of the cumulative distribution function, $\mathrm{S}(t)=$ 
$1-F(t)$; the hazard function as the probability density function divided by the survivor function, $h(t)=f(t) / S(t)$; and the integrated hazard function, $H(t)$ as the integral of the hazard function from zero to $t$. Equivalently, $H(t)=-\log [S$ $(t)]$, which permits ready estimation from the data. ${ }^{7}$

This measure, $C_{\mathrm{OR}}(t)$, employs the standard parallel model (recall: parallel, unlimited capacity, and with stochastically independent channels) as a kind of yardstick. If $C_{\mathrm{OR}}(t)=1$ for all values of $t$, performance is identical to that of the standard parallel model. That is, $C_{\mathrm{OR}}(t)$ values of $1 \mathrm{imply}$ that the system has unlimited capacity, such that processing in a given channel is not affected by the increase in workload due to the increase in the number of targets; that is, a given channel has the same processing rate whether a target is presented to another channel or not. Furthermore, $C_{\mathrm{OR}}(t)$ values that are below 1 imply that capacity is limited, such that increasing the processing load (by increasing the number of targets on the display) takes a toll on performance. Those limitations, when viewed through the "eye" of a parallel system, reveal performance that would entail from parallel channels, which slow down as workload increases. Finally, if $C_{\mathrm{OR}}(t)>1$, the system is said to have super capacity and a parallel interpretation indicates that processing efficiency of individual channels actually increases with increased work load. Of course, there are a number of different ways in which a system might produce limited or super capacity. Also, other models may exist that mimic the unlimitedcapacity signature of the standard parallel model. In theory, even a serial model might elicit exactly $C(t)=1$, but that seems highly unlikely in natural systems.

An increasing set of studies have used both the race model inequality and the capacity coefficient to describe the properties (capacity, independence vs. coactivation) of the system under investigation (e.g., Townsend \& Nozawa, 1995; Wenger \& Townsend, 2004b; and more recently, Berryhill, Kverga, Webb, \& Hughes, 2007; Eidels, Townsend, \& Algom, 2010b; and Eidels, Townsend, \& Pomerantz, 2008). Although related (cf. Townsend \& Wenger, 2004b), these measures are not identical and are generally presented separately. ${ }^{8}$ Here, we show how the race model inequality

\footnotetext{
${ }^{7}$ Logarithmic transformations of survivor functions were used by Mordkoff and Yantis (1991) and by Mulligan and Shaw (1980; although they looked at the marginal probabilities of "no" responses, not as a function of time) to test the independent-decision capacitysharing model. This model is mathematically identical to the standard parallel model that serves as a yardstick for the $C(t)$ index.

${ }^{8}$ From remarks at conferences and, occasionally, reviewer's commentaries, we feel there is some confusion about the optimal roles of the inequalities and the $C(t)$ functions. Sometimes, it is claimed that one is "superior" to the other. Actually, we believe that each plays a distinct and complementary part to the other. The bounds aid the scientist in obtaining a view of more or less extreme behavior; or not. They cannot assay workload efficiency in a continuous way as can the $C(t)$ functions, with the standard parallel performance acting as a yardstick. Also see the Discussion section.
}

can be mapped onto the capacity coefficient space. Thus, the two measures can be conveniently displayed on the same plot and compared with each other.

Townsend and Nozawa (1995) showed that the capacity coefficient can also be written using survivor functions. Substituting $H(t)=-\log [S(t)]$ into Eq. 2,

$$
C_{O R}(t)=\frac{-\log \left[S_{A B}(t)\right]}{-\log \left[S_{A}(t)\right]-\log \left[S_{B}(t)\right]}=\frac{\log \left[S_{A B}(t)\right]}{\log \left[S_{A}(t) \cdot S_{B}(t)\right]}
$$

This is important because we can then write the race model inequality in terms of survivor functions and, hence, in terms of the capacity coefficient, $C_{\mathrm{OR}}(t)$ (see Appendix 1 for the full derivation). ${ }^{9}$ Thus, an alternate but mathematically equivalent expression for the race inequality is

$C_{O R}(t) \leq \frac{\log \left[S_{A}(t)+S_{B}(t)-1\right]}{\log \left[S_{A}(t) \cdot S_{B}(t)\right]}$

This bound is illustrated in the top right panel of Fig. 1 as a dotted line. Observed $C_{\mathrm{OR}}(t)$ values that exceed the line violate the race model inequality. The solid line, at $C_{\mathrm{OR}}(t)=1$, corresponds to unlimited capacity (and thus marks the prediction of our yardstick standard parallel model). Note that when the race model bound is viewed in terms of the capacity function, it is, when defined, always higher than 1 (it approaches 1 when $t$ approaches 0 but increases sharply further away from 1 as $t$ increases - such that it becomes increasingly difficult to violate), implying that the race model bound is a conservative estimate of super capacity (cf. J. Miller, 1991; Townsend \& Wenger, 2004b). In other words, a processing system may have super capacity (i.e., $C_{\mathrm{OR}}(t)>1$ ), and yet these $C_{\mathrm{OR}}(t)$ values may still lie below the race model bound, not violating it.

\section{The Grice inequality (OR tasks)}

There is a bound on limited capacity (as opposed to the race model bound on super capacity) proposed by Grice and colleagues (Grice et al., 1984):

$F_{A B}(t) \geq \operatorname{MAX}\left[F_{A}(t), F_{B}(t)\right]$

If this inequality is violated, the system is limited capacity in a quite strong sense. In this case, performance on doubletarget trials $\left[F_{\mathrm{AB}}(t)\right]$ is slower than on those single-target trials that contain the faster of the two targets, $\operatorname{MAX}\left[F_{\mathrm{A}}(t)\right.$, $\left.F_{\mathrm{B}}(t)\right]$. In the top left panel of Fig. 1, the Grice bound is represented by the cumulative distribution function of the faster of the two single-target conditions (in this example, it

\footnotetext{
${ }^{9}$ Of course, the reverse is also possible: One can write (and plot) the capacity coefficient and the various bounds in terms of cumulative distribution functions. We leave this as an exercise to the readers.
} 
is B). The inequality is satisfied as long as the observed distribution of the double-target condition (thick solid line) stays above this line. By converting Grice's bound to its survivor function form, we can map it onto the $C_{\mathrm{OR}}(t)$ space. Doing so, we allow all pertinent tests (capacity coefficient, race model bound, and Grice's bound) to be parsimoniously presented on a single plot.

There is more than just convenience in this way of presentation. The relations between the capacity measure and the two bounds then reveal which of the assays are more conservative (or more liberal) and to what extent. The interpretation of the observed capacity coefficient values may be aided by the simultaneous presentation of the race model and Grice's bounds, and vice versa.

As for the race model inequality, the Grice bound can be rewritten as a function of $C_{\mathrm{OR}}(t)$ :

$C_{O R}(t) \geq \frac{\log \left\{M I N\left[S_{A}(t), S_{B}(t)\right]\right\}}{\log \left[S_{A}(t) \cdot S_{B}(t)\right]}($ see Appendix 1$)$

In the top right panel of Fig. 1 the Grice bound is represented by the dash-dot line. The bound is satisfied as long as the observed $C_{\mathrm{OR}}(t)$ function (thick solid line) stays above this line. Notice that this line marks poor performance to increased load, since it is below $C_{\mathrm{OR}}(t)=1$ for all $t$.

\section{AND task}

As was pointed out earlier, AND tasks demand the processing of all displayed items. Take, for example, the redundant-target search task described earlier, with four possible displays: target $\mathrm{A}$ alone, target $\mathrm{B}$ alone, targets $\mathrm{AB}$, or no targets at all. Participants are asked to respond affirmatively if and only if $\mathrm{A}$ and $\mathrm{B}$ both appear (the double-target display). Thus, in order to respond correctly, they have to exhaustively process all items. After the thorough presentation of the OR quantities, the AND paradigm can be treated rather expeditiously.

The Colonius-Vorberg inequalities and the AND capacity coefficient

Colonius and Vorberg (1994) proposed upper and lower bounds appropriate for AND tasks, analogous to the Miller and Grice bounds in the OR paradigm:

$F_{A}(t)+F_{B}(t)-1 \leq F_{A B}(t) \leq \operatorname{MIN}\left[F_{A}(t), F_{B}(t)\right]$

Here, $F_{\mathrm{AB}}(t)$ denotes $F_{\operatorname{MAX}\left(T_{A}, T_{B}\right)}(t)$ and refers to the cumulative distribution function of the double-target condition in the AND task [note that $F_{\mathrm{AB}}(t)$ refers to the cumulative distribution function of $\max \left(T_{\mathrm{A}}, T_{\mathrm{B}}\right)$ in the AND case, but to the distribution function of $\min \left(\mathrm{T}_{\mathrm{A}}, \mathrm{T}_{\mathrm{B}}\right)$ in the OR case]. Because processing has to be exhaustive, completion time on each trial is given by the completion time of the slower of two (or more) processes. Townsend and Wenger (2004b) developed a capacity measure appropriate for AND decisions, written as

$C_{A N D}(t)=\frac{K_{A}(t)+K_{B}(t)}{K_{A B}(t)}=\frac{\log \left[F_{A}(t) \cdot F_{B}(t)\right]}{\log \left[F_{A B}(t)\right]}$

The function $K(t)$ is analogous to the integrated hazard function, $H(t)$. If we let $k(t)$ be equal to the density divided by the distribution function, $k(t)=f(t) / F(t)$, then it can be thought of as the conditional probability density that processing completed in just the last instant, given that it completes at or before $t$. In that sense, $k(t)$-also termed the reverse hazard function (Chechile, 2011) - is analogous to the hazard function, $h(t)$, which we defined earlier as $h(t)=$ $f(t) / S(t)$, or the probability that a process just completed, given that it had not completed before time $t . K(t)$ is then defined as the integral of $k(t)$ from $t$ to infinity in an analogous way to $H(t)$ being defined as the integral of $h$ ( $t$ ) from 0 to $t$. Furthermore, in analogy to $H(t), K(t)=\log$ $[F(\mathrm{t})]^{10}$

Note that in our $C_{\mathrm{AND}}(t)$ index (Eq. 8), the single-target quantities are in the numerator, unlike the $C_{\mathrm{OR}}(t)$ index (Eq. 3). This inversion results in a common way to interpret $C_{\mathrm{AND}}(t)$ and $C_{\mathrm{OR}}(t)$ : Values greater than 1 imply performance superior to that of a standard parallel model; less than 1 is inferior to a standard parallel model; and values equal to 1 imply that performance is identical to that of a standard parallel model.

One of our reviewers asked whether it is necessary to define different capacity measures for the OR and AND tasks. A distinctive definition of capacity for the AND case is absolutely necessary since the stopping rule plays a critical role in the measurement of workload capacity. Basically, the exhaustive AND rule adds more time to the process over and above that required for the OR rule, even though there may be no change in the channels' processing rates in the two. This fact compels differences in the capacity formulas. Thus, it would be "unfair" to call a model for the AND task more limited in capacity than that for the OR task, simply because the former requires more channels to be completed. Note, though, that apart from the distinct decision rule for OR and AND cases, the yardstick model for both capacity measures is the same standard parallel model. For the mathematical detail, we point the

\footnotetext{
${ }^{10}$ We are grateful to Richard Chechile and Trish Van Zandt for noticing that our original definition stated that $K(t)$ was the integral of $k\left(t^{\prime}\right)$ from 0 to $t$, which is an error. We should have stated that $K(t)$ is $(-1)$ times the integral of $k\left(t^{\prime}\right)$ from $t$ to infinity. Fortunately, none of the theorems or inferences were harmed by this oversight.
} 
reader to Appendix 2 and other sources (e.g., Neufeld, Townsend, \& Jette, 2007; Townsend \& Wenger, 2004b).

We now show how to present different performance measures on a unified AND capacity space. Combining Eqs. 7 and 8 , we can express $F_{\mathrm{AB}}(t)$ in terms of $C_{\mathrm{AND}}(t)$ and, consequently, express the Colonius-Vorberg bounds on the $C_{\mathrm{AND}}$ space:

$\frac{\log \left[F_{A}(t) \cdot F_{B}(t)\right]}{\log \left[F_{A}(t)+F_{B}(t)-1\right]} \leq C_{A N D}(t) \leq \frac{\log \left[F_{A}(t) \cdot F_{B}(t)\right]}{\log \left\{\min \left[F_{A}(t), F_{B}(t)\right]\right\}}$

In Table 1 we summarize the pertinent inequalities for both the OR and the AND tasks, in their conventional and transformed forms. The various tests are expressed in terms of $F(t)$ and $S(t)$, so they can be conveniently estimated from experimental data.

\section{Model predictions}

In this section, we present, on the unified $C_{\mathrm{OR}}$ and $C_{\mathrm{AND}}$ spaces, simulation results from two models: (1) the standard parallel model - that is, a two-channel independent parallel model with unlimited capacity - and (2) the coactive model realized by parallel Poisson counters feeding into a final common channel.

Model predictions for OR paradigm: standard parallel model

The present instantiation of the standard parallel model assumes two independent channels, with unlimited capacity, each acting as a Poisson counter (e.g., Smith \& Van Zandt, 2000; see also Townsend \& Ashby, 1983, and Vickers, 1979, for general treatments of counting models). Evidence (measured in counts) toward a detection response is accumulated separately on each channel, until a prescribed criterion is met. In an OR task, processing time on a double-target condition is simply the time for the faster channel to complete its processing - that is, the time it takes for the number of counts in the faster channel to reach the criterion value. Mathematically, this model can be written as $S_{A B}(t)=S_{A}(t) \cdot S_{B}(t)$, which is just a formal way of saying that the probability that a response has not been made by time $t$ given two targets is the product of the probabilities that a response has not been made by time $t$ given either single target alone (Mordkoff \& Yantis, 1991, p. 535; Townsend \& Wenger, 2004b, p. 1013). With this definition at hand, we can calculate and plot the predictions of the standard parallel model for the OR case.

Processing latencies on each channel of this model are Gamma distributed, with specified rate and criterion values.
We explored a wide range of parameter values. In the following example, however, we assumed an equal criterion value (of 5 counts) for each of the channels. Rate values for channels $\mathrm{A}$ and $\mathrm{B}$ were set to .2 and .25 , respectively. ${ }^{11}$ Figure 1 shows the prediction of this model in the conventional form of cumulative distribution functions (top left panel) and in the novel form, mapped onto the $C_{\mathrm{OR}}$ space (top right panel). $C_{\mathrm{OR}}(t)$ values on the top right panel are exactly 1 . When we report the noisier empirical results, we address the issue of sample noise by plotting error bounds.

Model predictions for OR paradigm: Coactive model

This model too assumes two parallel channels. Unlike a race model, however, information from the two channels converges downstream to satisfy a single criterion. Hence, the evidence accumulation rate in a coactive Poisson model is the sum of rates of the individual channels. In this example, we assumed the same criterion value (5) and processing rates for the single channels $(.2, .25)$ as before. The processing rate for the double-target distribution is then given by summing the rates $(0.45)$. In the bottom row of Fig. 1, we present the prediction of a coactive model with respect to the pertinent bounds in the conventional form (bottom left panel) and in the novel form, mapped onto the $C_{\mathrm{OR}}$ space (bottom right panel). $C_{\mathrm{OR}}(t)$ values on the bottom right panel are above 1 , and the race model inequality is violated at almost all $t$ values, suggesting that capacity is super to a rather strong degree. This result is compatible with the simulations of Townsend and Wenger (2004b) and the analytic results of Townsend and Nozawa (1995).

Model predictions for AND paradigm: standard parallel model and coactive model

In the upper row of Fig. 2, we present predictions of a twochannel independent-parallel model with unlimited capacity (standard parallel model), which indicate the expected unlimited capacity. This model is identical to the standard model specified earlier for the OR case, except for the decision rule. To obtain this plot we used Gamma distributions with the same parameters as in the OR example (i.e., criterion $=5$, rate $\mathrm{A}=.2$, rateB $=.25$ ). The distribution function for the double-target condition in the AND case is given by $F_{A B}(t)=F_{A}(t) \cdot F_{B}(t)$ (e.g., Townsend \& Nozawa, 2004b, p. 1015), which means that the probability that a response has been made by time $t$ given

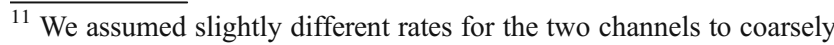
mimic the pattern observed in our empirical results (see Figs. 3 and 4).
} 
Fig. 2 Predictions of a standard parallel model (top row) and a coactive model (bottom row) in the AND task. The data with respect to the Colonius-Vorberg $(\mathrm{C}-\mathrm{V})$ bounds are presented on the left panels in the conventional distribution form, where the dotted line marks the lower bound and the upper bound is given by the distribution function of the slower single-target conditions (A in this illustration). In the right panels, we present the same bounds and data, transformed onto the capacity coefficient space

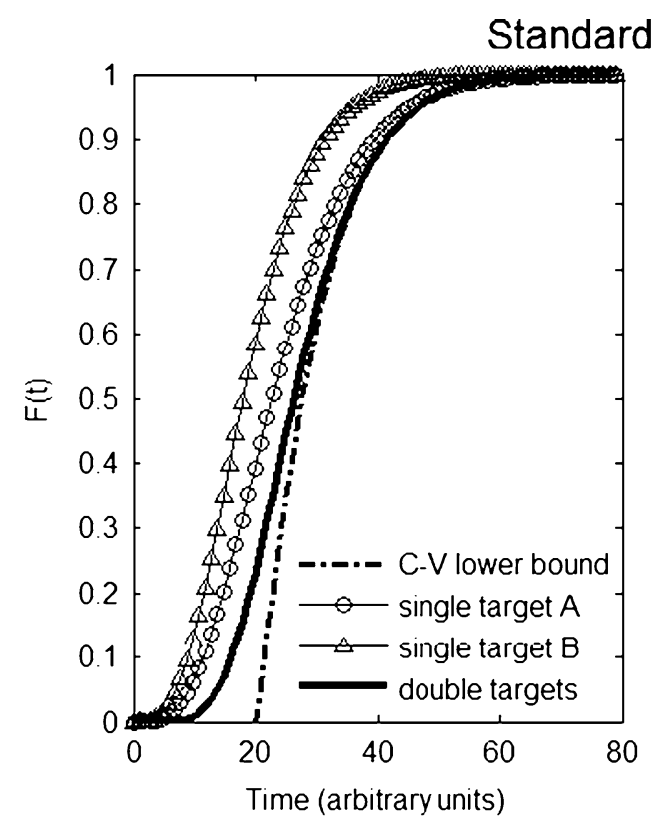

Standard Parallel Model
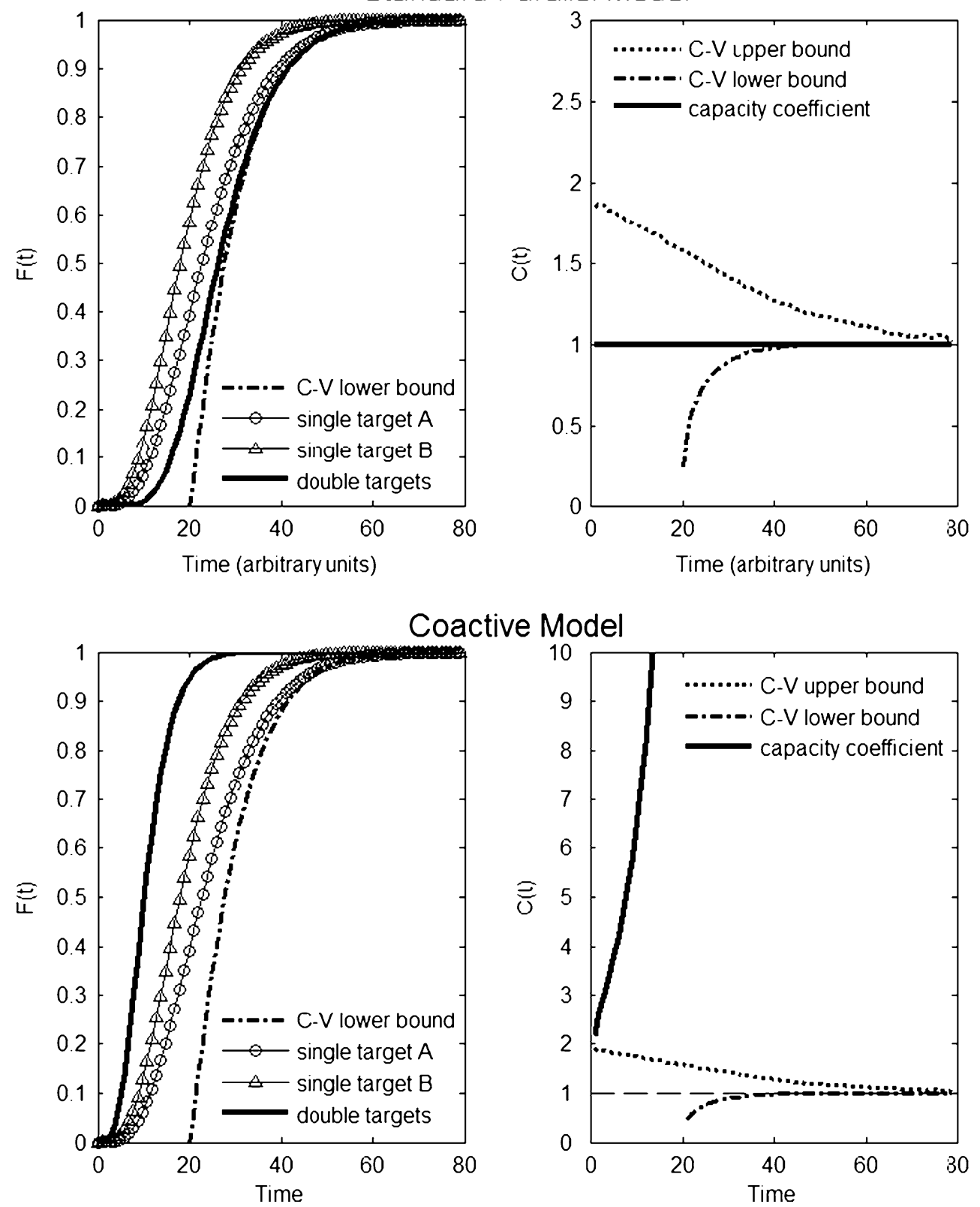

ive Model

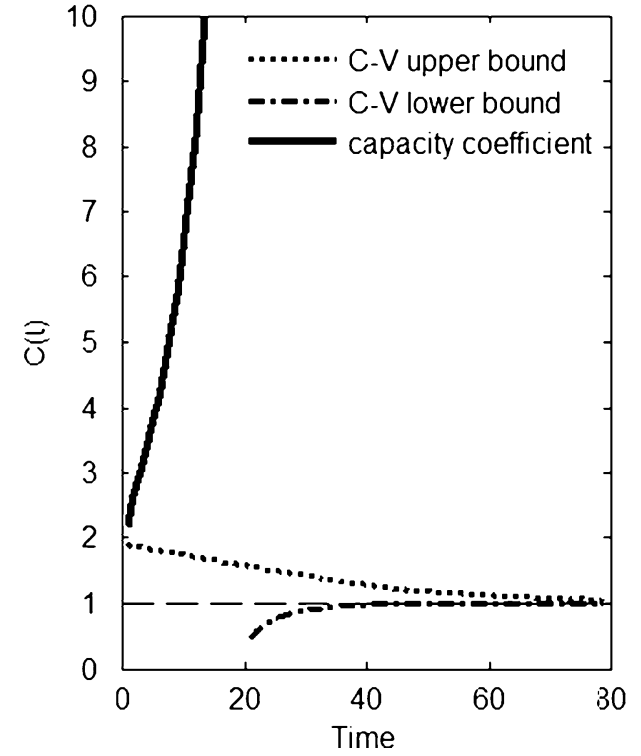

two targets is the product of the probabilities that a response has been made by time $t$ for both single-target A and singletarget $\mathrm{B}$. In the second row, we present predictions of a coactive Poisson counter model where, as in the OR case, the rate for the double-target distribution is the sum of single-target rates (.45). Dramatic super capacity is the result.

Following the simulations, we wished to perform a prototypical experiment to probe and illustrate the new procedures. We chose a modification of Townsend and Nozawa's (1995) design, which used dots as stimuli. Experiment 1 employed the OR task, as did the original study. Experiment 2 was an AND task, which has not previously been run with these stimuli.

\section{Experiment 1 (OR task)}

\section{Method}

The following experiment was performed in order to produce some exemplary data for illustrative purposes. The present data come from two highly trained participants, but the qualitative results, mainly parallelism, use of the optimal stopping rule (an OR decision), and modestly limited capacity (e.g., $C(t)<1$ and failure to violate either the Miller race bound above, or the Grice bound below), have so far described all participants; no individual differences were observed in the basic aspects of information 
processing in this task. ${ }^{12}$ These individuals participated, on consecutive days, in four experimental sessions (about an hour each) of a visual target detection task. The task was administered in a completely dark room, after $10 \mathrm{~min}$ of darkness adaptation.

In the task, a bright dot (luminance of $67 \mathrm{~cd} / \mathrm{m}^{2}$; subtending to $0.2^{\circ}$ of visual angle at a viewing distance of $50 \mathrm{~cm}$ ) could be presented (on a black screen) above a fixation point, below it, or on both positions or not presented at all. We refer to these targets as the top (A) and bottom (B) signals respectively. On a double-target display, two dots were displayed on a vertical meridian, equally spaced above and below a fixation point at an elevation of $\pm 1^{\circ}$. On a single-target display, only one dot was presented, either above or below fixation by $1^{\circ}$. The target-absent display consisted of a blank black screen. Stimuli were mixed within blocks.

The stimuli were generated via Microsoft Painter by an IBM-compatible (Pentium 4) microcomputer and displayed binocularly on a super-VGA 15 -in. color monitor with a $1,024 \times 768$ pixel resolution, using DMDX software (Forster \& Forster, 2003). On a trial, a single-pixel fixation point (subtending $0.05^{\circ}$ of visual angle at a viewing distance of $50 \mathrm{~cm}$; luminance of $0.067 \mathrm{~cd} / \mathrm{m}^{2}$ ) was presented on the center of the screen for $500 \mathrm{~ms}$, followed by a blank black screen $(500 \mathrm{~ms})$, and then followed by the stimulus display. The stimulus appeared on the screen for $100 \mathrm{~ms}$ and was then replaced by a blank screen. The participants were instructed to respond as quickly as possible. The response sampling began with the onset of the stimulus display and continued for $4,000 \mathrm{~ms}$. The intertrial-interval was $1,000 \mathrm{~ms}$. The participants were asked to respond affirmatively by pressing the right mouse key with the right index finger upon detection of at least one target (i.e., two dots, single dot on top, single dot at the bottom) and to respond no by pressing the left mouse key with the left index finger if no dot was present.

The probabilities of presenting both targets, presenting the top target alone, the bottom target alone, or no target at all were equal to .25. This means that the overall probability of a yes response in the OR task is .75 , and a response bias toward a positive response may ensue. However, the present methodology is insensitive to such biases, except insofar as, in principle, a contingency might be inadvertently set up so that information about the presence or absence in one location might inform about the same in the other location (Mordkoff \& Yantis, 1991). The .25 prior frequencies of presentations for all four

\footnotetext{
12 The one exception is that Experiment 2 of Townsend and Nozawa (1995) showed super capacity. The reason for this disparity is still unknown.
}

stimuli obviate the possibility of that kind of "correlation" (Mordkoff \& Yantis, 1991; Townsend \& Nozawa, 1995). In addition, the different statistics discussed here $\left[C_{\mathrm{OR}}(t)\right.$, race model inequality, and Grice inequality] are all based on trials from the same response (yes), so response bias, even if present, makes no difference to these statistics.

Each session started with a practice block of 100 trials, followed by five experimental blocks of 160 trials each, with a 2-min break between blocks. The order of trials was randomized within a block. Overall, a large number of trials, 3,200, were collected for each participant at each experiment (OR, AND), allowing for tests at the distributional level.

\section{Results}

We recorded and analyzed RTs from correct responses. Error rate was low (3.4\% and 2.2\% for participants 1 and 2, respectively) and no accuracy-RT trade-off was observed. Using the RTs, we estimated the cumulative distribution functions and other requisite statistics for each of the experimental conditions.

In Fig. 3, we present the relevant RT distributions and the pertinent tests. In the left panels, the cumulative distributions of single- and double-target conditions are presented against the conventional form of the race model bound. In the right panels, the same data are presented in the capacity space. Focusing on the right panels, both participants exhibited limited capacity, evident by capacity coefficient values that are consistently below 1 . Yet these capacity limitations were not severe enough to lead to violations of the lower, Grice bound. To better inform the reader about the stability of the estimated capacity function, we plot in thin solid lines the standard error of estimation (estimated by bootstrapping, with a correction to Van Zandt, 2002). ${ }^{13}$ Strong inferences about workload capacity can be made as long as the confidence interval is tight enough. For the time range where it was tight, the observed workload capacity is always below 1 for Participant 1

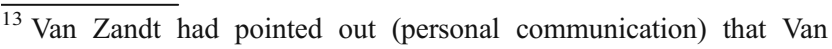
Zandt's (2002) method gives an underestimate of the true confidence interval, since it assumes that function values across time were independent. She proposed a method of expanding the confidence interval by some scaling factor, such that $90 \%$ of the function estimates fall within the expanded interval for all values of $t$. The confidence intervals presented in Figs. 2 and 4 as thin solid lines are, of course, the corrected, scaled intervals. The scaling factors for our OR and AND data are 1.99 and 2.04 (participant 1) and 2.20 and 2.17 (participant 2), respectively. This means that the true confidence bounds, according to this method, are about twice as far from the estimated $C(t)$ function, as compared with the estimates of the original method.
} 
Fig. 3 Experimental results from a target detection OR task. Data from participant 1 (top row) and participant 2 (bottom row) are presented against the conventional form of the Grice and the race model bounds (left panels) and on the capacity coefficient space (right panels). The thin solid lines above and below the capacity coefficient function, in the right panels, represent \pm 1 standard error of the estimate (estimated by bootstrapping; see Footnote 13)
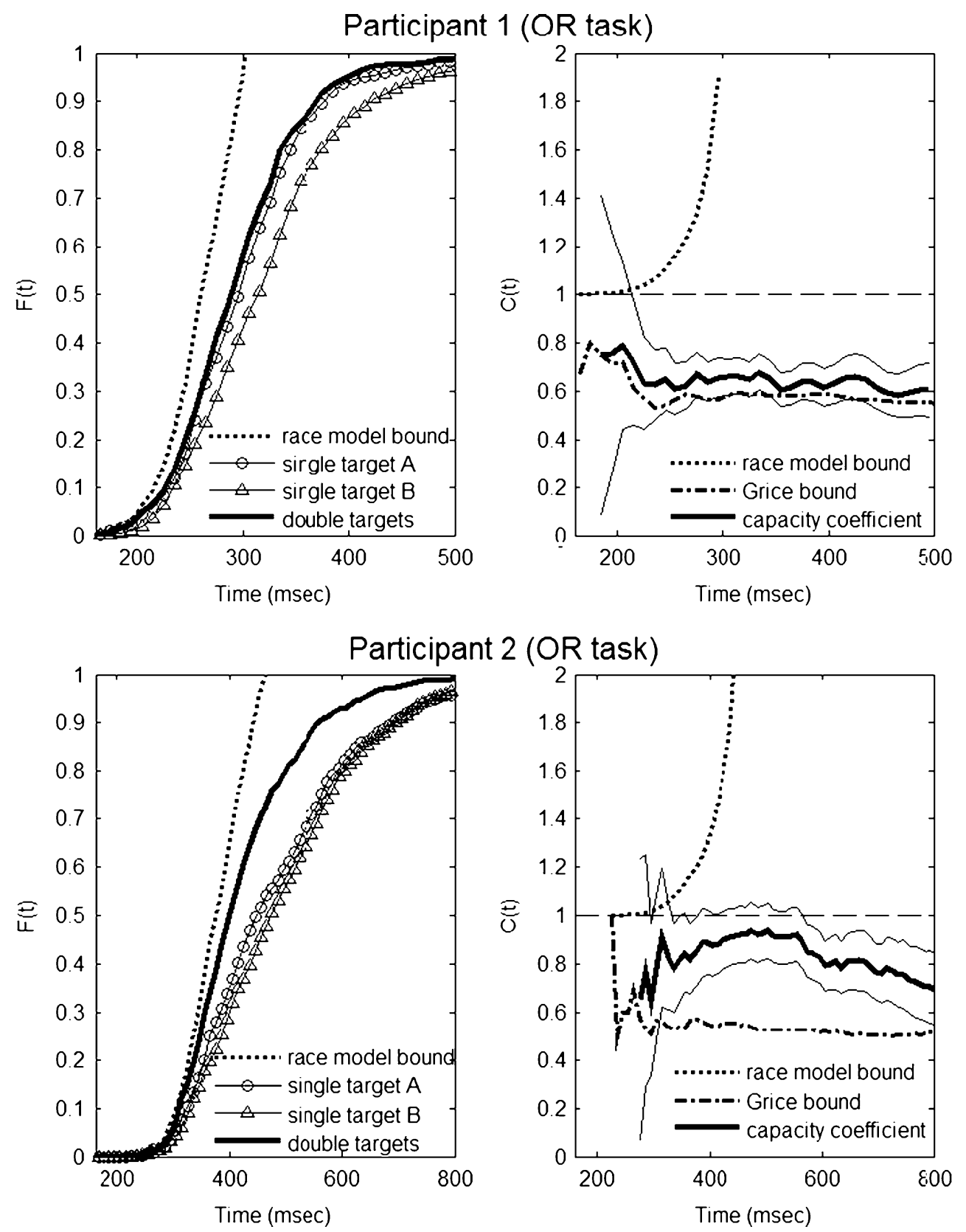

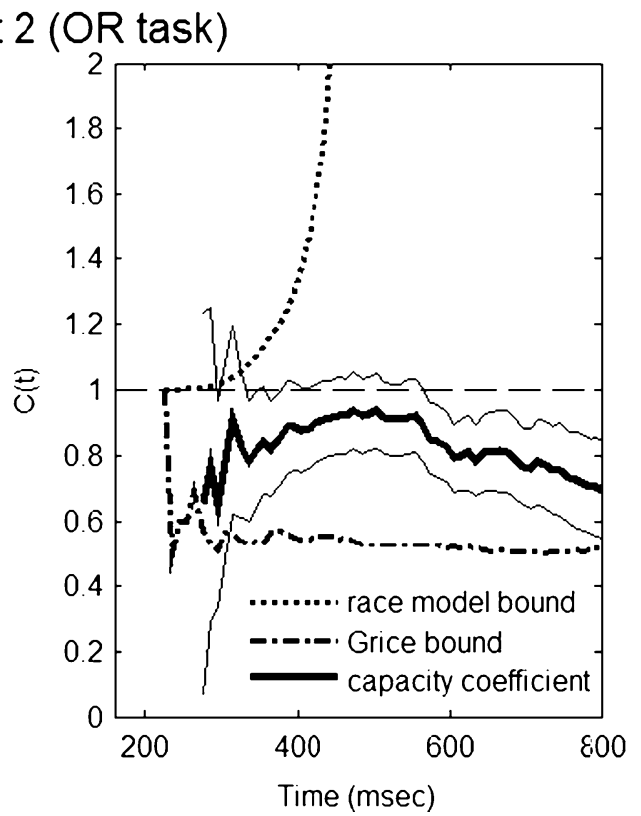

(anywhere between 230 and $500 \mathrm{~ms}$ ) and is either below or roughly bounded by 1 for participant 2 (anywhere between 330 and $800 \mathrm{~ms}$ ).

Therefore, models that predict super capacity, such as coactive models, can be rejected immediately, and for participant 1, even the standard parallel model is disconfirmed. In principle, coactive models with severe capacity limitations, or with a negative cross talk between processing channels can produce $C(t)<1$. However, Townsend and Wenger (2004b) showed that the benefits of coactivation are robust enough to render this scenario unlikely. They simulated coactive systems with positive and negative interchannel dependencies and concluded that "... the presence of negative cross talk prior to pooling does not come close to off setting the advantage obtained by pooling the channels." (p. 1024). A parallel model with fixed, evenly shared capacity (i.e., a fixed amount of processing resources that are equitably shared between channels) and a standard serial model both predict $C_{\mathrm{OR}}(t) \approx .5$ (see Townsend \& Ashby, 1983, pp. 76-91), and can therefore also be rejected with confidence. The simplest processing model that accommodates the observed data is a parallel model with rather significant limitations in resources, yet not to the level of the Grice bound. Again, results are in close alignment with the capacity findings of Townsend and Nozawa (1995, Experiment 1). 


\section{Experiment 2 (AND task)}

\section{Method}

In this task, the same participants who earlier performed the OR task performed in four further sessions, with instructions to respond affirmatively if and only if targets $\mathrm{A}$ and $\mathrm{B}$ were simultaneously presented (i.e., if dots appear in both upper and lower positions). Otherwise, if no targets were present or if either target A or target B were presented alone, the participants had to respond negatively by pressing another key. Because the probabilities of presenting both targets, the top target alone, the bottom target alone, or no target at all were all equal to .25 (as in the OR task), this means that in the AND task the overall probability of a yes response was .25. All other experimental details were the same as those in the OR experiment.

There is a practical distinction, although not a theoretical one, between the estimated $C_{\mathrm{AND}}(t)$ and $C_{\mathrm{OR}}(t)$ functions. Recall that in the OR paradigm, the single-target data can be extracted from the yes single-target trial data. However, in the AND paradigm, the single-target trials require a no response. Residual processes not engaged in the target detection may alter the RTs associated with the single-target no responses. For example, Clark and Chase (1972) argued that negation responses may, in general, consume more time than affirmation responses. Given this logic, we have proposed (Eidels \& Townsend, 2009) that a more realistic estimate of $C_{\mathrm{AND}}(t)$ can be garnered by employing singletarget yes data from an OR paradigm. This is the strategy we take here.

\section{Results}

$\mathrm{C}_{\mathrm{AND}}(t)$ and the Colonius-Vorberg bounds are plotted in Fig. 4. Capacity, as gauged by the capacity coefficient and shown in the right panels of Fig. 4, was quite limited in the early ranges, although never quite violating the lower Colonius-Vorberg bound. For medium and longer RTs, capacity became super, eventually superseding the upper bound, indicating the emergence of extreme super capacity.

\section{General discussion}

Comparing results from the OR and AND data and methodological considerations

Participants in Experiments 1 and 2 engaged in OR and AND tasks, with exactly the same stimuli in each paradigm, allowing for a direct comparison of capacity across the two tasks. Capacity on the OR task was limited for all $t$ values (Fig. 3, right panels), whereas capacity on the AND task was super over an extensive range of RTs (Fig. 4, right panels).

The researcher might consider as one possible account of the difference in AND versus OR capacities that the way in which task demands could affect the mode of processing. Instructions to detect both $\mathrm{A}$ and $\mathrm{B}$ targets may push human participants toward unitization of several items into a single, holistic, representation, resulting in super capacity. In fact, there are now several sets of data suggesting that forcing conjunctions of display elements, rather than disjunctions, can provide an impetus toward super capacity.

In point of fact, Blaha and Townsend (2006) have shown that in a conjunctive categorization task, in which participants had to exhaustively search all items of a set in order to respond correctly, participants exhibited capacity values much higher than 1 after a few days of training. In contrast, when searching for one particular feature, capacity was limited even after several days of training. In addition, employing OR and AND designs with facial emotional features, Innes-Ker and Townsend (2003) also discovered super capacity with exhaustive AND processing but limited capacity in the OR counterpart. In a broad investigation of word and face perception, Wenger and Townsend (2006) also documented considerably more super capacity in their AND conditions. Nonetheless, the present dot stimuli seem extraordinarily simple in contemplating this explanation. The investigator might also be interested in following up on the very early period of limited capacity.

Recall that the negation responses in the AND paradigm induced us to select affirmation responses from the OR paradigm. (This should always be carried out on a withinparticipants basis.) We recomputed $C_{\mathrm{AND}}(t)$, using instead the negation single-target responses from that condition and found uniform super capacity. Thus, for the major range of times, either technique delivers the inference of super capacity. Nevertheless, since this strategy may inflate $C_{\mathrm{AND}}(t)$ due to slower no single-target responses, the experimenter may well prefer the yes single-target data (i.e., singletarget data from an OR experiment) in computing this capacity index.

RT influences from various aspects of the processing system and the yardstick

Multiple sources of information can, in principle, be processed serially, in parallel, or in some hybrid fashion (see, e.g., Schweickert, 1978; Schweickert \& Townsend, 1989). Additionally, they might be processed independently from one another or interact in different ways. The processing efficiency of the system across various levels 
Fig. 4 Experimental results from a target detection AND task. Data from participant 1 (top row) and participant 2 (bottom row) are presented against the conventional form of the Colonius-Vorberg $(\mathrm{C}-\mathrm{V})$ bounds (left panels) and on the capacity coefficient space (right panels). The thin solid lines above and below the capacity coefficient function, in the right panels, represent \pm 1 standard error of the estimate (estimated by bootstrapping; see Footnote 13)
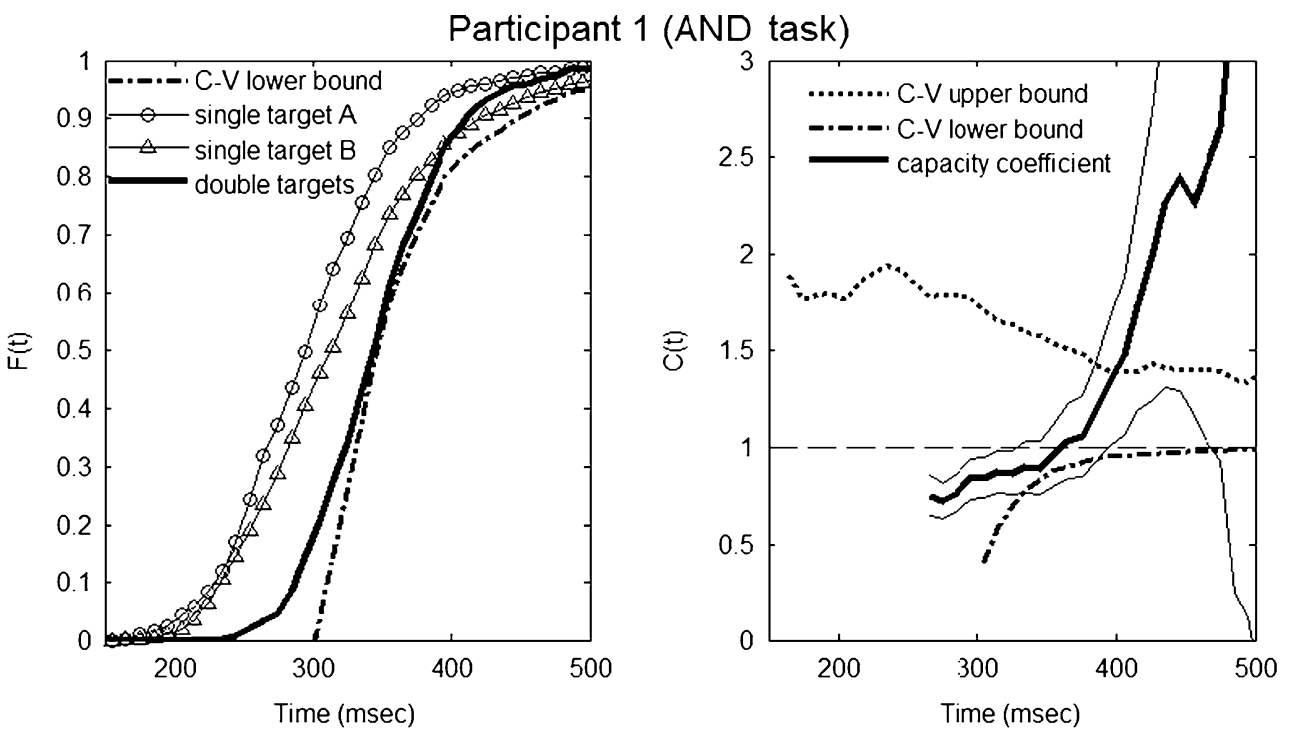
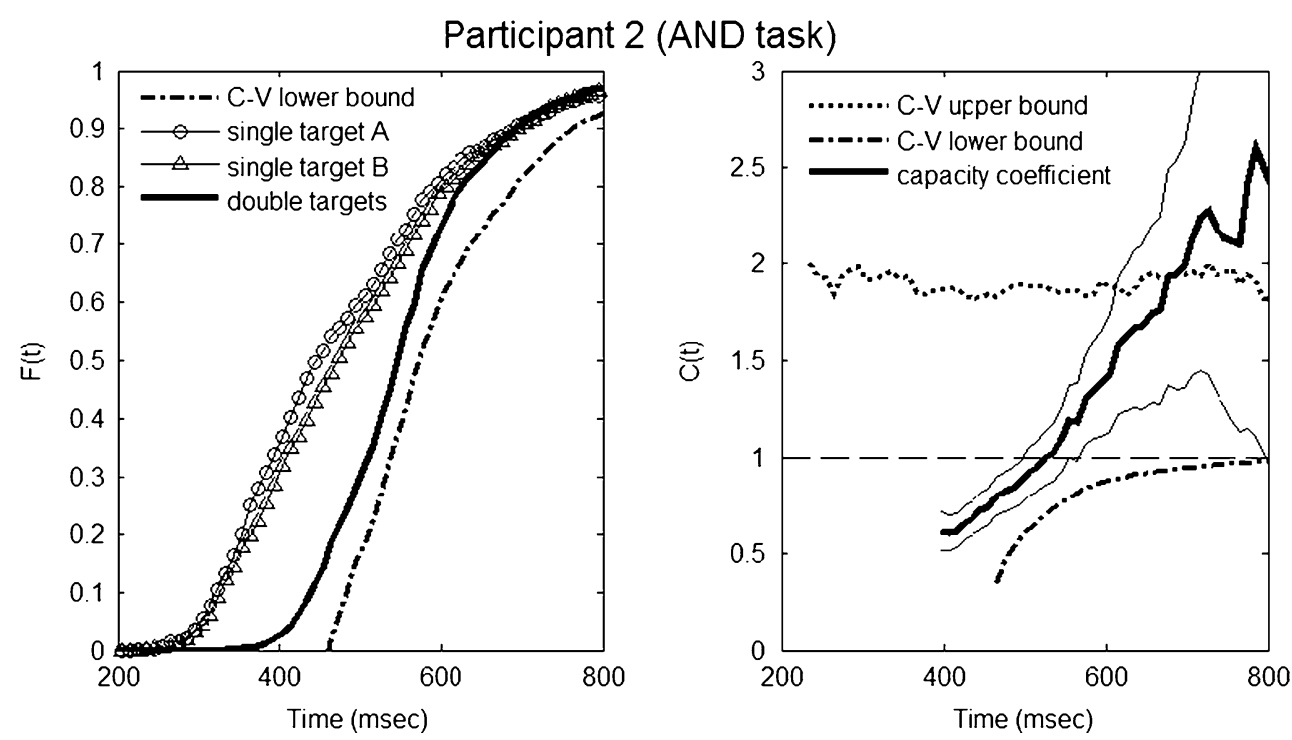

of workload can change as the effort required of the participant is varied. Naturally, the change can come about due to the architecture, for example, serial versus parallel, stopping rule, for example, OR versus AND tasks, channel or item dependencies, and so on.

Some commentators have been puzzled by the term workload capacity when it is used in the context of redundant targets in an OR task. The confusion apparently stems from the expectation that redundant OR responding will always be faster than, say, responding to either target separately. First, our capacity methodology assays the overall performance, which naturally compiles the contributions from both channels (stages, etc.). And our yardsticks allow, or take into account, the stopping rule. From that point of view, a system that is limited capacity in going from a single to double signal situation in an AND task will, with no further changes to the efficiency of the individual subprocesses, still be limited capacity in an OR situation. This fact is quite critical, since it will hold even if the limitations are sufficiently moderate that some benefits to the redundant target stimuli accrue (i.e., there is a redundancy gain, to use the parlance in the field of redundant signals). It is the underlying efficiency of the entire system that is being tested, not just the aspect of whether or not RT is shorter with two targets, rather than one.

Furthermore, it can be observed that a minimum-time serial system will not predict that a redundant-target trial is faster than a single-target trial (in fact, their averages should be the same). Finally, with regard to this particular issue, we have discovered stimuli and tasks where participants reveal capacity that is as poor as, or even poorer than, a fixed capacity (equivalently, minimum-time serial processing) parallel system. When workload capacity descends 
below the fixed capacity level, redundancy gains no longer occur. $^{14}$

The workload capacity measures we constructed are founded on the class of parallel systems in which the channels neither slow down nor speed up as the load is increased and, in addition, assume stochastic independence among the channels. The installation of this variety of parallel systems as the "yardstick" is somewhat subjective, and other investigators may think of good reasons to pick others - for instance, serial systems or coactive systems.

Our choice seems natural, and useful, to us for the following reasons. The parallel versus serial processing issue has surfaced in many diverse arenas and in a number of cases, the primary "antagonists" are what we refer to as standard serial models versus standard parallel models. The former class is based on one-at-a-time processing with identical, independent distributions for each completion time. The latter class is just that specified immediately above. The visual search literature forms one apposite example of this approach. Another imposing body of research largely pits race models against "something better," the something better usually being coactivation, which brings us to the next topic.

\section{Agreeing on a taxonomy of psychological systems}

The inductive side of our developments has primarily been drawn from the research spheres of visual and memory search and the redundant-signals literature. As is usually the case in psychological theory and methodology, the terminology has been rather vague in the past in either area. It presently seems important to establish conventions upon which investigators can agree or, at least, take as a point of departure.

In particular, terms such as race model and coactivation have been somewhat blurrily defined and further changed over the years. Let's first take up the definition of race model, which has played such a vital role in the redundantsignals purview. Starting in the 1970 s, and generated primarily by J. Miller and his colleagues (e.g., J. Miller,

\footnotetext{
${ }^{14}$ An easy, intuitive example is found with an exponential parallel model. Suppose the rates for channels $\mathrm{A}$ and $\mathrm{B}$ are $V_{\mathrm{A}}(N=1)=$ $V_{\mathrm{B}}(N=1)=\mathrm{V}(N=1)$, where $N=1$ indicates that the number of activated processing-channels is 1 (e.g., only one spatial position is occupied by a signal that requires processing). That is, no across-channel (e.g., position) effects are present. Suppose next that $V_{\mathrm{A}}(N=2)=$ $V_{\mathrm{B}}(N=2)=V(N=2)=V(N=1) / a$, where $a>2 . N=2$ indicates that the number of activated processing-channels is now two. This model produces mean minimum RT (neglecting residual components) for a single target that is $\operatorname{RT}(N=1)=1 / V$, and for double targets that is RT $(N=2)=1 /(2 V / a)=a /(2 V)>1 / V=\mathrm{RT}(N=1)$, so performance is worse on the double targets than on the singles. Furthermore, as Townsend and Nozawa (1995) pointed out, fixed capacity parallel models (and the capacity-equivalent serial models) sit precisely on the Grice bound (which marks rather limited workload capacity).
}

1978), race models were operationally defined by models (presumably, parallel models) that did not violate Miller's race model inequality. What we call the standard parallel model is what past researchers used as a standard (often referred to, somewhat awkwardly, as probability summation). Miller's bound is trivially satisfied by these models, as he well realized, thus promoting the more general, but somewhat vague, definition of race.

Subsequently, researchers began to turn to more theoretical definitions based on parallel channels that fed into a final common pooled channel, whose activation decided the final decision. All the rigorous examples with which we are familiar assumed stochastically independent processing in the distinct channels up until the pooling operation. We shall derive our proposed set of conventions from the analysis of Colonius and Townsend (1997), who took into consideration the evolution of terminology.

Our taxonomy first defines the class of separate decisions parallel models as any parallel system where detection decisions are made in the distinct channels, perhaps prior (as in redundant-signals designs) to imposing a final logical decisional rule. The class of race models is then defined as separate-decisions parallel processing with a minimum-time stopping rule. Such models can violate any of the bounds when channel speeds are somehow changed across workload level-for instance, through channel interactions. However, if the marginal channel speeds are preserved when the load is changed, the resulting race models will obey the Miller and Grice inequalities (ditto for the AND bounds; see Colonius, 1990; Colonius \& Vorberg, 1994).

Coactive models are next defined in terms of systems containing early independent parallel channels followed by a pooling into a single channel where the ultimate decision is made. Of course, hybrid process models are also possible (Colonius \& Townsend, 1997). For instance, one can design a system with positively or negatively interactive parallel channels but that posits a final pooling stage, as in our coactive models. With extremely negatively interacting channels, a hybrid coactive system can exhibit overall limited capacity. Again, these are conventions. Some might prefer to classify any system with a final pooling conduit, as "coactive," even if the separate channels interact prior to the pooling.

Our chosen taxonomy bears the following consequences, among others. (1) Separate decisions parallel models predict super capacity when channel facilitation is allowed (Eidels et al., 2011; Townsend \& Wenger, 2004b). (2) In fact, separate decisions parallel models with mutual channel facilitation can devolve to true coactivation as a special case (Colonius \& Townsend, 1997). (3) Separate decisions parallel models can be limited capacity if their channels inhibit one another (Eidels et al., 2011; Townsend \& 
Wenger, 2004b). (4) Separate decisions models might, as noted above, therefore either violate, or not, the Miller race inequality. (5) Coactivation models are invariably super capacity and readily violate the race inequality, if the individual channels are not seriously affected when the workload increases (Townsend \& Wenger, 2004b). (6) This system of classification implies that the former operational definition through violation of the race inequality is no longer viable. (7) Within this proposed convention, models whose channels interact early on and later converge via pooling should, by convention, not be called "coactive," although they may be viable hybrid models in certain conditions.

Finally, some have objected to segregating coactive and separate-decisions models in our classification. The reason is that both are multichannel, prior to the pooling or channel detections, respectively. Our taxonomy does possess the merit of preserving and extending the vital distinctions that have evolved in the field of redundanttarget perception.

\section{Influence of base times on capacity measures}

A component of the observed RTs, which we call base time, is known to have a potential effect on the estimates of the capacity coefficient and the race model bound. Base time is a traditional term for that duration that is consumed by subsidiary mechanisms before or after the internal processing interval of interest, and it often is symbolized by the random variable $T_{0}$. Then the random variable for RT, is just RT $=T_{\mathrm{p}}+T_{0}$, where $T_{\mathrm{p}}$ is the random processing time of interest. Typically, $T_{0}$ includes motor time and early sensory coding. It is virtually always assumed that $T_{0}$ is stochastically independent of the processes under study and also impervious to experimental manipulations of the latter. Progress has been made in the assessment of $T_{0}$ influences on our capacity measures and bounds, which will aid the researcher assaying capacity using the present methodology.

Townsend and Nozawa (1995) pointed out qualitatively (no rigorous proof) that the numerator of $C_{\mathrm{OR}}(t)$ contains only one $T_{0}$ component, whereas the denominator contains two, which, in general, will cause a lower estimate of $C(t)$ from what it would be if we could insert only the actual processing times of concern into the formula. As it turns out, analogous deformations act on the various bounds, although Ulrich and Giray (1986; see also Colonius, 1990; Colonius \& Vorberg, 1994) showed that inclusion of the base time would not permit satisfaction of the Miller or Grice inequalities if the processing time distributions violated them in the absence of the base time.

Nonetheless, Townsend and Honey (2007) recently proved that, when considering the base time component in
RTs, the capacity coefficient and race model inequality both generate an underestimate of the processing capacity of a system. In other words, one may observe $C_{\mathrm{OR}}(t)$ values below 1 , where, in fact, the real capacity of the system is unlimited or even super. Likewise, they demonstrated that both a maximum violation of the race bound and the area of the violation will be statistically less. The reason is the presence of $T_{0}$ in both the single-target RTs, but only once in the double-target RTs. They also simulated standard parallel models possessing base times of various magnitudes and found that, although the standard parallel models predict capacity coefficient values of 1 , the inclusion of a base time component pushed $C_{\mathrm{OR}}(t)<1$.

In this article (see Appendix 3), we prove for the first time that in AND tasks, the presence of $T_{0}$ in both the single-target RTs, but only once in the double-target RTs, has an effect opposite to that in OR tasks. Namely, if the standard parallel model is in force, our estimate of AND capacity with base time is $C_{\mathrm{AND}}(t) \geq 1$, as opposed to the contrary result in the case of $C_{\mathrm{OR}}(t)$ ! We return to utilize this fact later. In addition, Appendix 3 reports simulation results and considers the implications of base times in the OR and AND tasks.

Townsend and Honey (2007) discussed their theoretical findings in OR paradigms with regard to the experimental literature and provisionally concluded that, for base times that appear to be in the ballpark of what has been estimated in the laboratory, inferences about performance through $C_{\mathrm{OR}}(t)$ and the bounds would not be seriously damaged. In both the OR and AND paradigms, the magnitude of the variance of $T_{0}$ versus that of the processing times of interest will be the determining factor, as Townsend and Honey showed in the OR case. The typical $T_{0}$ variance appears negligible relative to the variability in the processes under study. The upshot for present purposes is that a $C_{\mathrm{OR}}(t)>1$ result and a violation of Miller's race bound are then strong evidence for true super capacity, whereas very mild limited capacity might be due to the base time. Large departures of $C_{\mathrm{OR}}(t)$ below 1 indicate true limitations in capacity whether due to limited resources or lateral inhibition. Our present simulations suggest that a substantial $C_{\mathrm{AND}}(t)$ in conjunction with a violation of the upper Colonius-Vorberg bound will provide sturdy evidence for high super capacity but a small increase of $C_{\mathrm{AND}}(t)$ over $C_{\mathrm{AND}}(t)=1$ may be due to $T_{0}$. Likewise, values of $C_{\mathrm{AND}}(t)<1$ cannot be evoked by $T_{0}$.

The theoretical findings above come into play when the researcher contemplates that, for each of the observers, limited capacity was found in the present OR task but super capacity, for the most part, in the AND task. This asymmetry plainly opens the possibility to the researcher that the base time could be at least partly responsible. Thus, she/he should ask whether it is possible that the base time 
was responsible for the difference in OR and AND experimental results, by way of leading to underestimations of the capacity index in the former and overestimation in the latter. The conclusion to be reached from extensive simulations (Appendix 3) is that base time is a sufficient explanation for the moderately limited capacity found in the OR data but that it is insufficient to account for the substantial super capacity discovered in the AND data.

The discovery of the opposite directions of influence by the base time in the OR and AND designs appears to be a quite felicitous event, as suggested by its aid in resolving the OR-AND capacity asymmetry above. It implies that carrying out both OR and AND designs can, as in the present circumstance, aid the experimenter in evaluating whether apparent limited capacity in the OR task is due to base time, and analogously for super capacity in the AND task. It is beyond our present scope, but this property may also help in adjudicating best model comparisons when parameterized models are fit to data in the two paradigms.

The discussion above opens up the possibility that other factors - rather than channels' processing rates under varying loads - can drive our capacity measures up or down. Fitting parametric models to data can provide estimates, rather than assumptions, about such parameters and whether they vary with workload. In a recent study, Eidels, Donkin, Brown, and Heathcote (2010a) have analyzed data from a redundant-target OR task by using the nonparametric $C_{\mathrm{OR}}(t)$ measure, as well as by fitting the linear ballistic accumulator model (Brown \& Heathcote, 2008). They found close agreement between the techniques and, in particular, discovered that the efficiency of processing across load conditions (single vs. double target) was driven by channels' accumulation rates, and not by other parameters such as base time. For example, in a group of participants who exhibited super capacity, the withinchannel processing rate for double targets was estimated as substantially higher than the single-target rate, meaning that each channel actually performed better when the other channel was activated. Base times, however, were almost identical and could not account for the observed super capacity, in complete agreement with Townsend and Honey's (2007) conclusion.

Other factors, such as decision threshold, may also play a role in determining the speed of response. Indeed, Eidels et al. (2010a) estimated for the group of participants above a lower threshold value for yes trials, as compared with no trials, presumably because, in the standard OR design, $75 \%$ of the trials require a positive response. However, in the OR task, this difference cannot contaminate $C_{\mathrm{OR}}(t)$ estimates, since the index is calculated only on the basis of yes trials. Future research may probe the effects of decision threshold on $C_{\mathrm{AND}}(t)$.
Distinct roles for the $C(t)$ functions and the bounds in a global theory

Consider a prototypical target detection task (e.g., J. Miller, 1978). As we have seen, the workload capacity coefficient (Townsend \& Nozawa, 1995; Townsend \& Wenger, 2004b) measures the effects of workload (number of features, items, channels, and so forth to be processed) on performance. These $C(t)$ functions can operate within distinct stopping rules such as OR and AND paradigms. Miller's race model inequality and Grice's inequality place upper and lower bounds on the performance of parallel processing with a minimum-time (OR) stopping rule (J. Miller, 1978, 1982; Grice et al., 1984). Violation of the Colonius and Vorberg (1994) upper bound indicates a processing speed that is superior to that expected from a standard parallel model employing an exhaustive (AND) stopping rule, and their lower bound does the opposite below. Both types of assessment can readily be extended to arbitrary workloads (for bounds, see Colonius, 1990, and Colonius \& Vorberg, 1994; for capacity indices, see Blaha \& Townsend, 2006).

The question might occur to the reader as to whether both the $C(t)$ index functions and the various bounds are really needed. A related issue is whether one approach might be in some sense "better" than the other. ${ }^{15}$

These statistics bear distinct purposes and advantages and disadvantages, although we view them as mutually supportive. Violation of Miller's race model inequality permits rejection of a large class of race models, but not all, since reasonable parallel race models with cross-channel facilitation can readily violate that bound (Townsend \& Wenger, 2004b). Within our theory, it assesses whether capacity is super beyond the bound level. Similarly, violation of the Grice inequality indicates extremely limited capacity not ordinarily associated with race models (but possible through, for example, mutually inhibitory channels; Townsend \& Wenger, 2004b).

A limitation of the race model inequality is that it is only useful for $F_{\mathrm{A}}(t)+F_{\mathrm{B}}(t) \leq 1$. Furthermore, although the race bound is typically very close to $C_{\mathrm{OR}}(t)=1$ for small values of $t$, there is a rapid divergence of the bound as time becomes larger, as shown for the first time in the above exposition. The Grice bound is always substantially below $C_{\mathrm{OR}}(t)=1$.

The Colonius-Vorberg inequalities play an analogous role in AND designs. The lower Colonius-Vorberg bound is virtually equivalent to $C_{\mathrm{AND}}(t)=1$ for very slow responses. However, the divergence of the capacity coefficients for shorter values of time provides concrete evidence of the difference in the statistics. The upper Colonius-Vorberg bound is typically significantly removed from $C_{\mathrm{AND}}(t)=1$. Analogously to the fact that the (upper)

\footnotetext{
$\overline{15}$ These issues have repeatedly been raised in reviews and conversations, and never, so far as we know, in published articles.
} 
race bound is useful only when $F_{\mathrm{A}}(t)+F_{\mathrm{B}}(t) \leq 1$, the lower Colonius-Vorberg bound is appropriate only when $F_{\mathrm{A}}(t)+F_{\mathrm{B}}(t) \geq 1$.

We hope we have persuaded the reader that both methodologies contribute distinct but essential information and that they offer valuable complementary evidence. Indeed, both the capacity functions and the various bounds are contained in an overall general theory of capacity (Townsend \& Wenger, 2004b), which now permits contrast and comparison on the same scale. In the present article, we extend this general theory by developing a new commonscale framework. This framework affords the simultaneous depiction of all pertinent measures of performance employing a common $C(t)$ scale and in the same data space. Using the transformation formulae in Table 1 (presented in their $F$ $(t)$ and $S(t)$ form, so they can be easily computed from experimental data), one can plot, for instance, the lower Colonius-Vorberg alongside the prediction of a standard parallel model, $C_{\mathrm{AND}}(t)=1$, and experimental and/or simulated data on the same scale.

In closing, it is worth noting that although we have emphasized OR and AND experimental designs, our methods can be extended to any situation involving an arbitrary number of objects required to be processed in some manner. For instance, any task based on the sentential logical calculus will necessitate a finite number of elements to be processed. For instance, an XOR (exclusive OR) task- "respond positively if one signal is present, or another, but not both"-demands the determination of exactly one target and one nontarget on a yes trial, implying exhaustive processing.

Interestingly, stimuli containing two targets or no targets can also call for exhaustive processing. The present methodology for assessment of capacity, as well as that of architecture and stopping rule and dependence, can be generalized to such tasks.

\section{Conclusion}

This study proposes a conception of capacity spaces wherein not only can the original $C(t)$ indices be depicted, but also such other vital statistics as the Miller, Grice, and Colonius and Vorberg bounds can be graphed and contrasted. Naturally, experimental data and, perhaps, simulations of complex models whose capacity predictions may not be immediately evident can be graphed in such a space and compared with pertinent theoretical bounds. Although we have emphasized several varieties of parallel processing and the major inequalities, it is feasible to portray the predictions of other architectures-for instance, serial models or more complex architectures (e.g., Schweickert \& Townsend, 1989). In addition, it is perfectly feasible to chart, say, AND predictions, or data for that matter, within an OR capacity space. Thus, the AND predictions from a standard parallel model in such a portrayal reveal how much a system is "paying" by failing to implement a disjunctive, first-terminating, minimumtime decision strategy, but rather using an exhaustive, conjunctive rule.

Author Note This project was supported by NIH-NIMH MH 057717-07 and AFOSR FA9550-07-1-0078 research grants to J.T.T., and by the Keats Endowment grant to A.E. We greatly appreciate helpful commentaries from Richard Chechile, Trisha Van Zandt, and Dirk Vorberg.

\section{Appendix 1 Transformations to a common capacity space}

Here, we show how to express the race model inequality and the Grice inequality on the capacity coefficient space. We begin by recalling the mathematical expression for the capacity coefficient (Townsend \& Nozawa, 1995):

$C_{O R}(t)=\frac{H_{A B}(t)}{H_{A}(t)+H_{B}(t)}$

Expressing the race model inequality on the capacity space

The race model inequality (J. Miller, 1978, 1982) is given by

$F_{A B}(t) \leq F_{A}(t)+F_{B}(t)$

We can write the race model inequality in terms of survivor functions. By substituting $F(t)$ with $[1-S(t)]$ and rearranging we get

$S_{A B}(t) \geq S_{A}(t)+S_{B}(t)-1$

Townsend and Nozawa (1995) showed that the capacity coefficient can also be written using survivor functions, so we can express $C_{\mathrm{OR}}(t)$ in terms of $S_{\mathrm{AB}}(t)$ :

$C_{O R}(t)=\frac{-\log \left[S_{A B}(t)\right]}{-\log \left[S_{A}(t)\right]-\log \left[S_{B}(t)\right]}=\frac{\log \left[S_{A B}(t)\right]}{\log \left[S_{A}(t) \cdot S_{B}(t)\right]}$

And, conversely, can express $S_{\mathrm{AB}}(t)$ in terms of $C_{\mathrm{OR}}(t)$ :

$S_{A B}(t)=\left[S_{A}(t) \cdot S_{B}(t)\right]^{C_{O R}(t)}$

Finally, by substituting $S_{\mathrm{AB}}(t)$ with the expression on the right hand side of Inequality 12 , we get

$S_{A}(t)+S_{B}(t)-1 \leq\left[S_{A}(t) \cdot S_{B}(t)\right]^{C_{O R}(t)}$ 
After rearranging, this gives the race model inequality expressed in terms of the capacity coefficient, $C_{\mathrm{OR}}(t)$ :

$C_{O R}(t) \leq \frac{\log \left[S_{A}(t)+S_{B}(t)-1\right]}{\log \left[S_{A}(t) \cdot S_{B}(t)\right]}$

Practically, this means that the race model inequality can be estimated from empirical data and plotted as a line on the capacity space (as shown by the upper dotted line in the right panel of Fig. 1). If values of the empirical $C_{\mathrm{OR}}(t)$ function exceed this line (at any $t$ for which inequality 16 is defined), then the inequality is violated.

Expressing the Grice inequality on the capacity space

The Grice inequality (Grice et al., 1984) is given by

$F_{A B}(t) \geq \operatorname{MAX}\left[F_{A}(t), F_{B}(t)\right]$

We convert Inequality 17 to its survivor function form, which, after rearranging, gives

$S_{A B}(t) \leq \operatorname{MIN}\left[S_{A}(t), S_{B}(t)\right]$

Then, by substituting $S_{\mathrm{AB}}(t)$ with the expression on the right hand side of Equation 14, we eventually get Grice's inequality expressed in terms of the capacity coefficient, $C_{\mathrm{OR}}(t)$ :

$C_{O R}(t) \geq \frac{\log \left\{\operatorname{MIN}\left[S_{A}(t), S_{B}(t)\right]\right\}}{\log \left[S_{A}(t) \cdot S_{B}(t)\right]}$

Like the race model inequality, the Grice inequality can be estimated from empirical data and plotted as a line on the capacity space (this time shown by the lower dotted line in the right panel of Fig. 1). For the inequality to hold, values of the capacity coefficient should be greater than or equal to the expression on the right hand-side of Inequality 19. If values of the empirical $C_{\mathrm{OR}}(t)$ function are found below this line, then the inequality is violated.

Note that if we know which of the two single targets $(\mathrm{A}, \mathrm{B})$ is processed faster, we can reduce this inequality to a much simpler form. If, for instance, we know that target B is processed faster, then the inequality becomes $C_{O R}(t) \geq \frac{\log \left[S_{B}(t)\right]}{\log \left[S_{A}(t) \cdot S_{B}(t)\right]}$. However, to avoid loss of generality it is useful to leave Inequality 19 as it is.

\section{Appendix 2 Distinguishing AND from OR capacity: The technical facts}

As was mentioned in the text, a new definition of capacity for the AND case is absolutely necessary since the stopping rule plays a critical role in the measurement of workload capacity. Let us explain this point by using the definition of the standard parallel (unlimited capacity, independent, and parallel) model. This model is the benchmark against which we calculate the efficiency of processing in our $C(t)$ measures. For the AND case, the model is defined by $F_{A B}(t)=F_{A}(t) \cdot F_{B}(t)$, where $F_{\mathrm{AB}}(t), F_{\mathrm{A}}(t)$, and $F_{\mathrm{B}}(t)$ are the cumulative distribution functions for RTs in the doubletarget $(\mathrm{AB})$ and single-target (A alone, $\mathrm{B}$ alone) conditions. For the system to finish processing and correctly detect the presence of both signals, it must wait for both channel A and channel $\mathrm{B}$ to complete. Because we assume independence, the joint probability of completion at or before time $t$ in channels $\mathrm{A}$ and $\mathrm{B}$ is the product of completion probabilities in each channel alone. Now, for the OR case, the model is easily defined by the probabilities of noncompletion. If detecting a signal in any of the channels is sufficient to evoke a response, then the probability of noncompletion given a two-targets display is the product of noncompletion probabilities in each channel: $\left[1-F_{A B}(t)\right]=$ $\left[1-F_{A}(t)\right] \cdot\left[1-F_{B}(t)\right]$, which can be also written in terms of survivor functions: $S_{A B}(t)=S_{A}(t) \cdot S_{B}(t)$. It is clear from this exercise that the standard parallel model is defined differently for different stopping rules (AND, OR). Note, in Eqs. 8 and 3 , that the $C_{\mathrm{AND}}(t)$ and $C_{\mathrm{OR}}(t)$ measures are logarithmic transformations of these definitions. Our $C_{\mathrm{AND}}(t)$ and $C_{\mathrm{OR}}(t)$ compare performance against the same type of model, but the exact mathematical definition of this model depends on the stopping rule. Clearly, our workload capacity calculations must also differ depending on the stopping rule.

\section{Appendix 3 The standard parallel model and assay of base time influences}

Here, we first establish that probabilistically, the presence of a base time causes the capacity estimate in an AND design to appear greater than the true capacity. Interestingly, this is just the opposite of what occurs in OR designs (cf. Townsend \& Honey, 2007). This reversed role of the base time can be of assistance in assessing the magnitude of its influence and its relative contribution in the OR vs. AND paradigms. Then we perform a series of simulations that collectively indicate that although a moderately sized base time could account for the modest limitation in OR capacity, even significantly larger base times cannot produce the degree of super capacity found in the AND design.

Recall that the prediction of a standard parallel model that excludes base time is $C_{\mathrm{AND}}(t)=1$. Next, we show that the prediction of a standard parallel model with base time is $C^{*}{ }_{\mathrm{AND}}(t) \geq 1$. Suppose that $\mathrm{RT}_{\mathrm{A} \text {,and }}=T_{\mathrm{A}}+T_{0}, \mathrm{RT}_{\mathrm{B} \text {,and }}=$ $T_{\mathrm{B}}+T^{\prime}{ }_{0}$, and $\mathrm{RT}_{\mathrm{AB} \text {,and }}=\operatorname{MAX}\left(T_{\mathrm{A}}, T_{\mathrm{B}}\right)+T^{\prime \prime}{ }_{0}$, where $T_{\mathrm{A}}$, $T_{\mathrm{B}}$, and $T_{\mathrm{AB}}$ are the random processing times of interest and the different $T_{0} \mathrm{~S}$ are the random variables for base time. 
The tick marks on the $T_{0}$ random variables simply indicates that although they are all distributed the same, the experiment would sample independent values of it. Then, the following relationship holds for the distribution functions of a standard parallel model with base time (Townsend \& Honey, 2007, p.256; see also Colonius, 1990):

$F_{M A X\left(T_{A}, T_{B}\right)+T_{0}^{\prime \prime}}(t) \geq F_{T_{A}+T_{0}}(t) \cdot F_{T_{B}+T_{0}^{\prime}}(t)$

Taking the natural log of both sides and dividing by the expression on the left, we reverse the inequality sign (since

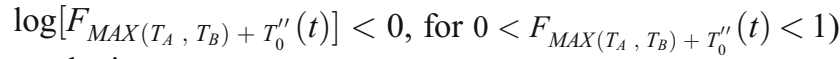
to obtain

$\frac{\log \left[F_{T_{A}+T_{0}}(t) \cdot F_{T_{B}+T_{0}^{\prime}}(t)\right]}{\log \left[F_{M A X\left(T_{A}, T_{B}\right)+T_{0}^{\prime \prime}}(t)\right]} \geq 1$

Compare the expression on the left hand-side of Eq. 21 with Eq. 8 and observe that the former is actually the capacity coefficient formula for the standard parallel model with base time (for the AND case), which we designate $C^{*}{ }_{\mathrm{AND}}(t)$. Because $C^{*}{ }_{\mathrm{AND}}(t) \geq 1$, and since the
Fig. 5 Simulated results from a standard parallel model with base time. Data from an OR task are presented in the top row, and data from an AND task are at the bottom. The data and bounds are presented in the left panel in their conventional form and transformed onto the capacity coefficient space in the right panel. The prediction of a standard parallel model without considering base time is represented by the dashed line at $C(t)$ $=1$ (regardless of whether it is an OR or an AND task)
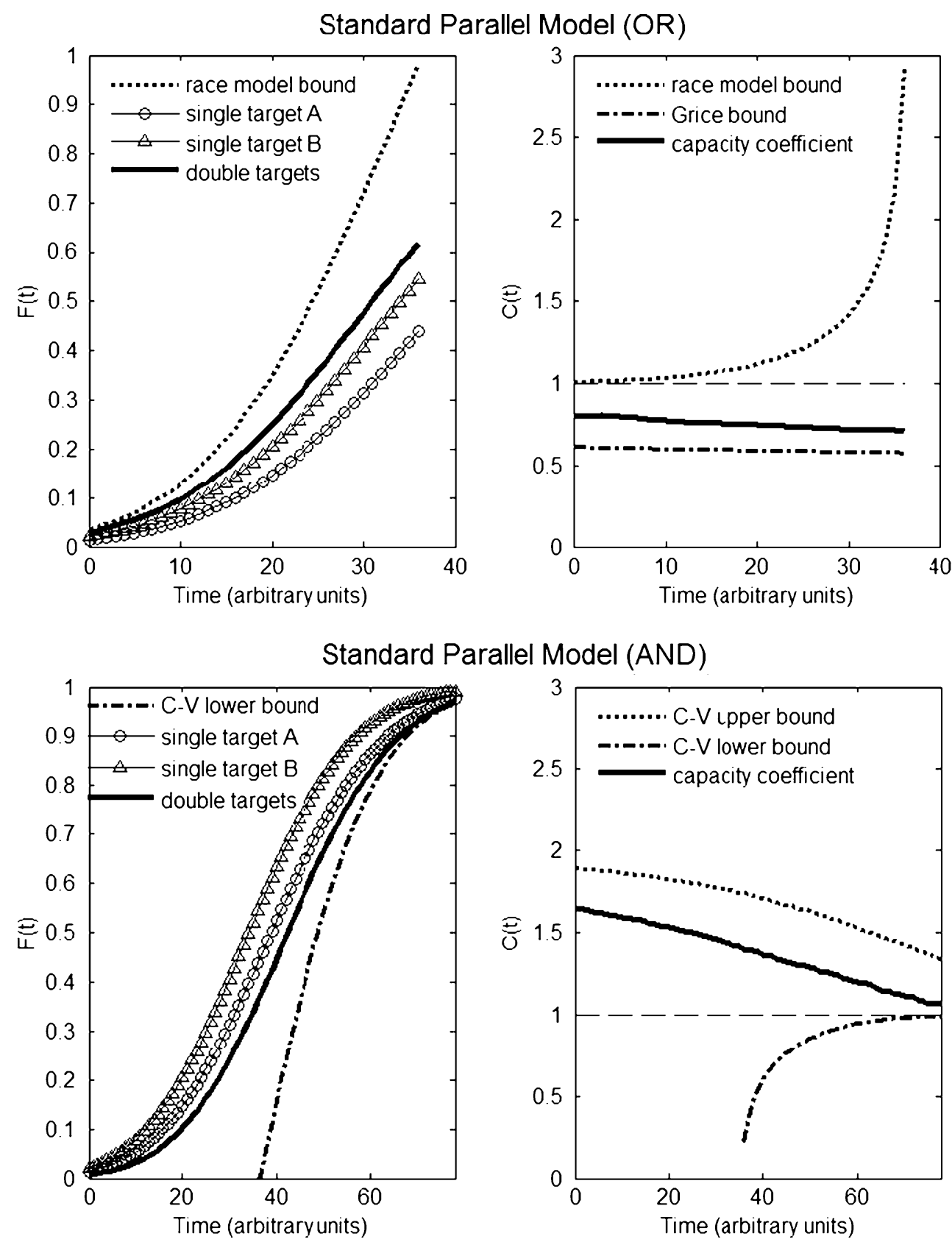

\section{arallel Model (AND)}

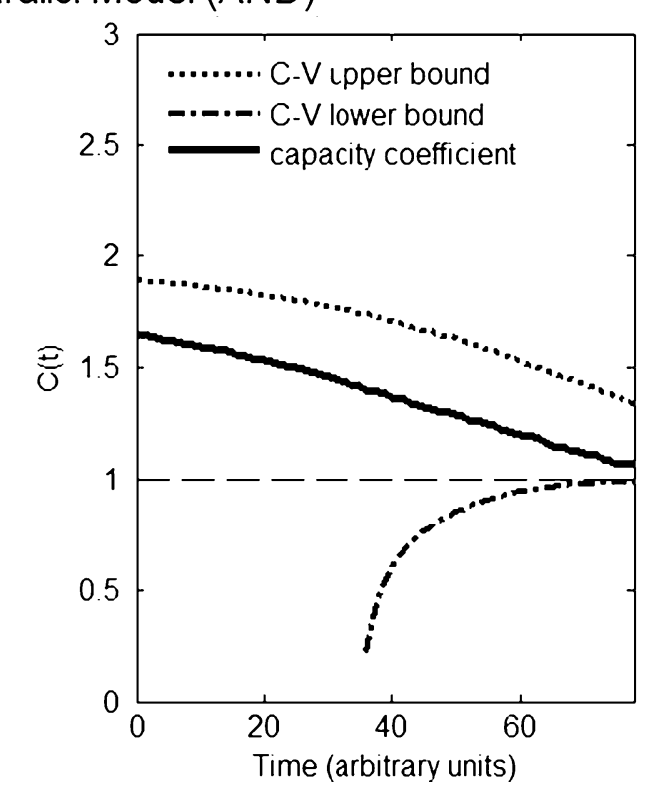


prediction of a standard parallel model without base time is $C_{\mathrm{AND}}(t)=1$, then $C^{*}{ }_{\mathrm{AND}}(t) \geq C_{\mathrm{AND}}(t)$, meaning that the presence of a base time causes the capacity estimate in an AND design to appear greater than the true capacity of the system.

In order to further assess the implications of base time on the capacity coefficient values in the new $C$ spaces, we simulated standard parallel models (i.e., parallel, unlimited capacity and independent channels) that include a base time. For each parallel channel, we sampled at random 150,000 values from independent Gamma distributions, with the same parameter values mentioned earlier in the text. In the OR task, the processing time of double targets, on each trial, is given by the processing time of the fastest single target - that is, by the minimum of the processing times of each single target. In the AND task, the processing time of double targets, on each trial, is given by the maximum of the processing times of each single target. We then added, for each process and on each trial, a base time component drawn at random from a normal distribution with a mean of 15 and a standard deviation of 15 , then estimated the distribution functions and recalculated the capacity coefficient.

The results are presented in Fig. 5. Simulated data from an OR task are presented on the top row, and simulated data from an AND task are at the bottom. Although the standard parallel model predicts capacity coefficient values of 1 , the inclusion of a base time component pushed $C_{\mathrm{OR}}(t)$ below 1 in the OR case - in fact, to estimated $C_{\mathrm{OR}}(t)$ values that hover, on average, around .7-much like the bulk of the observed data. Qualitatively in agreement with the AND data, capacity was pushed above unity there. However, the same parameters that produced behavior close to the OR results were unable to account for the extent of super capacity observed in the AND task or the early portion of the $C_{\mathrm{AND}}(t)$ function that was limited capacity.

The magnitude of the biasing effect caused by base time to the estimates of capacity can vary. Townsend and Honey (2007) showed that for the OR case, the magnitude of this bias increases with the proportion of the total RT variance contributed by base time. We therefore simulated a standard parallel model with a range of base time variance levels to test its effect on the estimates of capacity in the AND case. Figure 6 shows that $C_{\mathrm{AND}}(t)$ for a standard parallel model increases as we increase the standard deviation of the base time component (from 0 to 15 , in steps of 1). Nonetheless, even the rather extreme standard deviations probed in our simulations were unable to reach the high levels of super capacity found in portions of the $C_{\mathrm{AND}}(t)$ curve, much less the limited capacity part of the curve shown in Fig. 4.

The conclusion here is that base time is a sufficient explanation for our moderately limited capacity found in

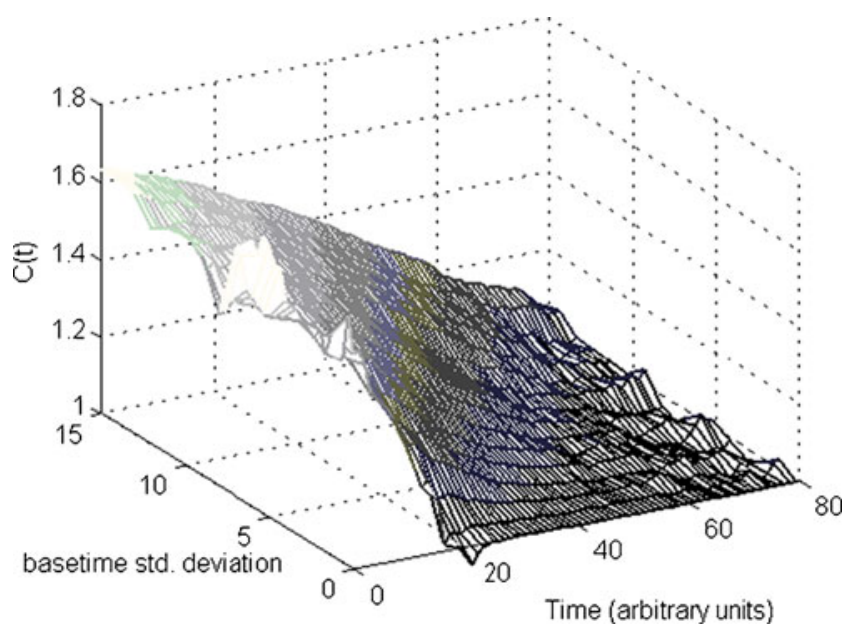

Fig. 6 Simulated results from a standard parallel model with base time (AND task). $C_{\mathrm{AND}}(t)$ estimates gradually increase as the variance of base time (represented here by the standard deviation) increases. The prediction of a standard parallel model without base time is $C_{\mathrm{AND}}(t)=1$

the OR data. However, an intriguing and sizable variation of AND capacity values from limited to super is inexplicable on account of a base time component and must rely on some other explanation, such as those expressed in the text. Very few AND experiments have been carried out employing our new capacity coefficient; it should be of considerable interest and assistance in identifying underlying processing structures to delve further into the effects of stopping rule on efficiency.

\section{References}

Ashby, F. G., \& Townsend, J. T. (1986). Varieties of perceptual independence. Psychological Review, 93, 154-179.

Baddeley, A. D. (2001). Is working memory still working? American Psychologist, 56, 851-864.

Baddeley, A. D., \& Hitch, G. J. (1974). Working memory. In G. A. Bower (Ed.), Recent advances in learning and motivation, Vol. 8 (pp. 47-90). New York: Academic Press.

Berryhill, M., Kverga, K., Webb, L., \& Hughes, H. C. (2007). Multimodal access to verbal name codes. Perception \& Psychophysics, 69, 628-640.

Blaha, L. M., \& Townsend, J. T. (2006, May). Parts to wholes: Configural learning fundamentally changes the visual information processing system. Paper presented at the Annual Meeting of the Vision Sciences Society, Sarasota, FL

Brown, S. D., \& Heathcote, A. (2008). The simplest complete model of choice reaction time: Linear ballistic accumulation. Cognitive Psychology, 57, 153-178.

Busey, T. A., \& Townsend, J. T. (2001). Independent sampling vs. inter-item dependencies in whole report processing: Contributions of processing architecture and variable attention. Journal of Mathematical Psychology, 45, 283-323.

Chechile, R. A. (2003). Mathematical tools for hazard function analysis. Journal of Mathematical Psychology, 47, 478-494. 
Chechile, R. A. (2011). Properties of the reverse hazard functions. Journal of Mathematical Psychology (in press).

Clark, H. H., \& Chase, W. G. (1972). On the process of comparing sentences against pictures. Cognitive Psychology, 3, 472-517.

Colonius, H. (1990). Possibly dependent probability summation of reaction times. Journal of Mathematical Psychology, 34, 253275.

Colonius, H., \& Townsend, J. T. (1997). Activation-state representation of models for the redundant-signals-effect. In A. A. J. Marley (Ed.), Choice, decision and measurement: Essays in honor of R. Duncan Luce (pp. 245-254). Mahwah: Erlbaum.

Colonius, H., \& Vorberg, D. (1994). Distribution inequalities for parallel models with unlimited capacity. Journal of Mathematical Psychology, 38, 35-58.

Cowan, N. (2005). Working memory capacity. New York, NY: Psychology Press.

Diederich, A., \& Colonius, H. (1991). A further test of the superposition model for the redundant-signals effect in bimodal detection. Perception \& Psychophysics, 50, 83-86.

Eidels, A., Donkin, C., Brown, S. D., \& Heathcote, A. (2010a). Converging measures of workload capacity. Psychonomic Bulletin \& Review, 17, 763-771.

Eidels, A., Houpt, J. W., Altieri, N., Pei, L., \& Townsend, J. T. (2011). Nice guys finish fast and bad guys finish last: Facilitatory vs. inhibitory interaction in parallel systems. Journal of Mathematical Psychology, 55, 176-190.

Eidels, A., \& Townsend, J. T. (2009, August). Testing response time predictions of a large class of parallel models within $O R$ and $A N D$ redundant signals paradigm. Paper presented at the 42ndAnnual Meeting of the Society of Mathematical Psychology, Amsterdam.

Eidels, A., Townsend, J. T., \& Algom, D. (2010). Comparing perception of Stroop stimuli in focused versus divided attention: Evidence for dramatic processing differences. Cognition, 114, $129-150$.

Eidels, A., Townsend, J. T., \& Pomerantz, J. R. (2008). Where similarity beats redundancy: The importance of context, higherorder similarity, and response assignment. Journal of Experimental Psychology: Human Perception \& Performance, 34, 14411463.

Forster, K. I., \& Forster, J. C. (2003). DMDX: A Windows displayprogram with milliseconds accuracy. Behavior Research Methods, Instruments, \& Computers, 35, 116-124.

Grice, G. R., Canham, L., \& Gwynne, J. W. (1984). Absence of a redundant-signals effect in a reaction time task with divided attention. Perception \& Psychophysics, 36, 565-570.

Innes-Ker, A.H., \& Townsend, J.T. (2003, November). Gestalt perception of emotional expressions. Paper presented at the 44thAnnual Meeting of the Psychonomic Society, Vancouver.

Kahneman, D. (1973). Attention and effort. Englewood Cliffs: Prentice Hall.

Lu, Z. L., \& Dosher, B. A. (1998). External noise distinguishes attention mechanisms. Vision Research, 38, 1183-1198.

Luce, R. D. (1986). Response Times: Their role in inferring elementary mental organization. New York: OxfordUniversity Press.

Miller, G. A. (1956). The magical number seven, plus or minus two: Some limits on our capacity for processing information. Psychological Review, 63, 81-97.

Miller, J. (1978). Multidimensional same-different judgments: Evidence against independent comparisons of dimensions. Journal of Experimental Psychology: Human Perception and Performance, 4, 411-422.

Miller, J. (1982). Divided attention: Evidence for coactivation with redundant signals. Cognitive Psychology, 14, 247-279.
Miller, J. (1991). Channel interaction and the redundant-targets effect in bimodal attention in bimodal divided attention. Journal of Experimental Psychology: Human Perceptions and Performance, 17, 160-169.

Miller, J., \& Ulrich, R. (2003). Simple reaction time and statistical facilitation: A parallel grains model. Cognitive Psychology, 46, $101-151$.

Mordkoff, J. T., \& Yantis, S. (1991). An interactive race model of divided attention. Journal of Experimental Psychology: Human Perception and Performance, 17, 520-538.

Mulligan, R. M., \& Shaw, M. L. (1980). Multimodal signal detection: Independent decisions vs. integration. Perception \& Psychophysics, $28,471-478$.

Neufeld, R. W. J., Townsend, J. T., \& Jette, J. (2007). Quantitative response time technology for measuring cognitive-processing capacity in clinical studies. In R. W. J. Neufeld (Ed.), Advances in clinical cognitive science: Formal modeling and assessment of processes and symptoms (pp. 207-238). Washington, DC: American Psychological Association.

Palmer, J., Verghese, P., \& Pavel, M. (2000). The psychophysics of visual search. Vision Research, 40, 1227-1268.

Parzen, E. (1962). Stochastic processes. San Francisco: Holden Day.

Schwarz, W. (1994). Diffusion, superposition, and the redundant target effect. Journal of Mathematical Psychology, 38, 501-520.

Schweickert, R. (1978). A critical path generalization of the additive factor method: Analysis of a Stroop task. Journal of Mathematical Psychology, 18, 105-139.

Schweickert, R., \& Townsend, J. T. (1989). A trichotomy method: Interactions of factors prolonging sequential and concurrent mental processesin stochastic PERT networks. Journal of Mathematical Psychology, 33, 328-347.

Shannon, C. E. (1948). A mathematical theory of communication. Bell System Technical Journal, 27(379-423), 623-656.

Shannon, C. E., \& Weaver, W. (1949). The mathematical theory of communication. Urbana: University of Illinois Press.

Shaw, M. L. (1982). Attending to multiple sources of information: I. The integration of information in decision making. Cognitive Psychology, 14, 353-409.

Smith, P. L., \& Van Zandt, T. (2000). Time-dependent Poisson counter models of response latency in simple judgment. British Journal of Mathematical and Statistical Psychology, 53, 293-315.

Sternberg, S. (1966). High-speed scanning in human memory. Science, 153, 652-654.

Townsend, J. T. (1972). Some results concerning the identifiability of parallel and serial processes. British Journal of Mathematical and Statistical Psychology, 25, 168-199.

Townsend, J. T. (1974). Issues and models concerning the processing of a finite number of inputs. In B. H. Kantowitz (Ed.), Human information processing: Tutorials in performance and cognition (pp. 133-168). Hillsdale, NJ: Erlbaum.

Townsend, J. T. (1981). Some characteristics of visual whole report behavior. Acta Psychologica, 47, 149-173

Townsend, J. T. (1990). Truth and consequences of ordinal differences in statistical distributions: Toward a theory of hierarchical inference. Psychological Bulletin, 108, 551-567.

Townsend, J. T., \& Ashby, F. G. (1978). Methods of modeling capacity in simple processing systems. In J. Castellan \& F. Restle (Eds.), Cognitive theory, Vol. III (pp. 200-239). Hillsdale: Erlbaum.

Townsend, J. T., \& Ashby, F. G. (1983). The stochastic modeling of elementary psychological processes. Cambridge: Cambridge University Press.

Townsend, J. T., \& Honey, C. J. (2007). Consequences of base time for redundant signals experiments. Journal of Mathematical Psychology, 51, 242-265. 
Townsend, J. T., \& Nozawa, G. (1995). Spatio-temporal properties of elementary perception: An investigation of parallel, serial, and coactive theories. Journal of Mathematical Psychology, 39, 321-359.

Townsend, J. T., \& Nozawa, G. (1997). Serial exhaustive models canviolate the race inequality: Implications for architecture and capacity. Psychological Review, 104, 595-602.

Townsend, J. T., \& Wenger, M. J. (2004a). The serial-parallel dilemma: A case study in a linkage of theory and method. Psychonomic Bulletin \& Review, 11, 391-418.

Townsend, J. T., \& Wenger, M. J. (2004b). A theory of interactive parallel processing: New capacity measures and predictions for a response time inequality series. Psychological Review, 111, 1003-1035.

Ulrich, R., \& Giray, M. (1986). Separate-activation models with variable base times: Testability and checking of cross-channel dependency. Perception \& Psychophysics, 39, 248-254.
Van Zandt, T. (2002). Analysis of response time distributions. In J. T. Wixted \& H. Pashler (Eds.), Stevens's Handbook of experimental psychology: Vol. 4. Methodology in experimental psychology (3rd ed., pp. 61-516). New York: Wiley.

Vickers, D. (1979). Decision processes in visual perception. London: Academic Press.

Wenger, M. J., \& Townsend, J. T. (2006). On the costs and benefits of faces and words: Process characteristics of feature search in highly meaningful stimuli. Journal of Experimental Psychology: Human Perception and Performance, 45, 283323.

Wenger, M. J., \& Gibson, B. S. (2004). Using hazard functions to assess changes in processing capacity in an attentional cuing paradigm. Journal of Experimental Psychology: Human Perception and Performance, 30, 708-719. 